\title{
A hyperelastic-bilinear potential for lattice model with fracture energy
}

\section{conservation}

\author{
Zhennan Zhang ${ }^{1 *}$, Jiafeng Ding ${ }^{1}$, Ahmad Ghassemi ${ }^{2}$, Xiurun $\mathrm{Ge}^{1}$ \\ ${ }^{1}$ School of Naval Architecture, Ocean and Civil Engineering, Shanghai Jiao Tong University, \\ Shanghai, 200240, China \\ ${ }^{2}$ Mewbourne School of Petroleum and Geological Engineering, University of Oklahoma, Norman, \\ OK 73091, USA
}

\begin{abstract}
:
A hyperelastic-bilinear potential (HBP) is proposed for lattice model to preserve the fracture energy in fracture simulation. This potential inherits the essence of hyperelastic interatomic potential in that it can capture the hyperelastic behaviors of material at the crack tip which plays a critically important role in dynamic fracture. Moreover, the potential retains the essence of the bilinear cohesive law in that it can preserve the fracture energy by setting a limit on the bond strain. With this HBP, the lattice size sensitivity is eliminated to a great extent and the dynamic fracture can be more accurately simulated.
\end{abstract}

Key words: Hyperelastic-bilinear potential; Lattice model; Fracture energy; Dynamic fracture simulation

${ }^{*}$ Corresponding author. Tel./fax: +86 2134204346.

Email address: zhennanzhang@sjtu.edu.cn (Z. Zhang) 


\begin{tabular}{|c|c|}
\hline \multicolumn{2}{|c|}{ Nomenclature } \\
\hline$A$ & physical parameter of bond potential \\
\hline$B$ & shape parameter of bond potential \\
\hline$E$ & Young's modulus \\
\hline$f_{t}$ & tensile strength of bond \\
\hline$G_{f}$ & initial fracture energy \\
\hline$G_{F}$ & total fracture energy \\
\hline$h$ & characteristic size of unit cell \\
\hline$h_{\text {critical }}$ & critical unit cell size \\
\hline$K_{I}^{c}$ & fracture toughness of material \\
\hline$\ell$ & bond length \\
\hline$\ell_{0}$ & bond length in un-deformed state \\
\hline$\ell_{b}$ & bond length at peak force \\
\hline$\ell_{p}$ & bond length at transition point of post-peak bilinear cohesive law \\
\hline$\ell_{r}$ & bond length corresponding to initial fracture energy \\
\hline$\ell_{c}$ & limit bond length over which bond breaks \\
\hline$L$ & length of the straight line segment intersected by the square \\
\hline$L_{1}, L_{2}, L_{3}$ & extended crack length at different time \\
\hline$n$ & total number of triangular unit cells cut by crack \\
\hline$N$ & number of bonds cut by crack facet in a unit cell \\
\hline$S$ & area of the created crack facet \\
\hline$S_{\text {exact }}$ & exact crack area \\
\hline$t$ & time \\
\hline$t_{0}$ & time to fracture \\
\hline$t_{1}$ & time of accelerated compact \\
\hline$\Delta t$ & time interval \\
\hline$v_{1}, v_{2}, v_{3}$ & crack speed at different time \\
\hline$V$ & volume of 3D unit cell and area of 2D unit cell \\
\hline$V_{0}$ & compact velocity \\
\hline$V_{1}$ & compact velocity constant \\
\hline$W_{1}$ & work contributed by the hyperelastic potential \\
\hline$W_{2}$ & work contributed by the first segment of post-peak linear cohesive law \\
\hline$W_{3}$ & work contributed by the second segment of post-peak linear cohesive law \\
\hline$\beta$ & bond force ratio \\
\hline$\varepsilon_{t}$ & uniaxial strain at the peak tensile stress of material \\
\hline$\tilde{\varepsilon}_{t}$ & bond strain at the peak force of bond \\
\hline$\Phi$ & hyperelatic bond potential \\
\hline$\gamma$ & coefficient of crack facet created in a unit cell \\
\hline$\theta$ & fracture energy ratio \\
\hline$\rho$ & material density \\
\hline$\sigma_{t}$ & uniaxial tensile strength of material \\
\hline$\sigma_{c}$ & uniaxial compressive strength of material \\
\hline$\tilde{\sigma}_{t}$ & simulated uniaxial tensile strength of material \\
\hline$v$ & Poisson ratio \\
\hline$\Omega$ & bond number in a unit cell \\
\hline
\end{tabular}




\section{Introduction}

Numerical methods for simulation of fracture can generally be divided into two broad categories namely, the continuum and the discrete methods. The continuum method considers material as a continuous medium and uses a constitutive relation to describe the mechanical response of the solid. Based on the constitutive relation, the crack tip stress field can be obtained through an analytical or a numerical method. The fracture propagation is predicted based on a certain criterion in terms of stress intensity factors. Because of their rigorous theoretical basis, the continuum methods generally are robust and powerful when dealing with the onset of fracture propagation. But once the fracture begins to extend, they face many challenging issues. For instance, to continue the calculation, the meshing scheme has to be updated if the finite element or boundary element method is used. Though some techniques are developed to avoid remeshing, e.g. the extended finite element method, the computation effort still is quite heavy. Another drawback of the continuum methods is that the constitutive relation used is unable to predict the complicated microscopic mechanisms, even though those mechanisms can have a strong influence on the macroscopic behavior [1].

The lattice method pioneered by Hrennikoff [2] is a type of discrete method, which represents a continuum using a discrete lattice system. It uses a simple formulation to intrinsically model the rupture phenomenon. Using this method, complex microscopic behaviors can be represented with a relatively small number of parameters. The macro fracturing process is simulated by the successive rupture of bonds. Therefore, the continuum fracture criterion in terms of stress intensity factors, and the complicated mesh generation problems that plague the continuum method are avoided. The lattice method has been extensively applied to concrete and many other disordered materials [3-7]. The lattice method is predominantly used to simulate the brittle fracture. But, recently, many attempts are devoted to considering the plastic effect, e.g.[8-11]. An important strength of the lattice method is that it transforms a 3D continuum fracture issue into an intrinsic $1 \mathrm{D}$ bond rupture problem. The $1 \mathrm{D}$ bond rupture mechanism is more straightforward and easier to characterize.

In spite of these advantages, the lattice method still has some drawbacks in fracture simulation. For instance, the regular lattice configuration has a preferential direction of fracture propagation [12-15]. The fracture trajectory obtained with such lattice configuration is sometimes a modeling artifact rather than the true behavior of material that modeled [16]. Monette and Anderson[14] suggested that the anisotropy of the failure surface could be responsible for the directional preference, and it can be remedied by the unstructured lattice configuration. Other scholars $[12,13$, 15,17 suggest the mesh dependency can be eliminated by randomizing the lattice configuration. Besides the regular configuration, the lattice type (spring, beam with two and three degrees of freedom per node) also has strong influence on the crack pattern[16]. The bias of fracture propagation induced by lattice type can also be circumvented by using a random lattice configuration according to [16]. Obviously, the random lattice configuration is a universal 
measure to remedy the mesh dependence problem. Meanwhile, the random lattice configuration can present various microstructure features, as well as a diverse oriented and localized phenomena. But, it cannot represent the uniform strain field.

The classical lattice models can simulate tensile fracture quite well, but they are inadequate when simulating the compressive cases. They cannot yield the corrected failure envelopes for triaxial compressive stress states. Under the hydrostatic compression condition, the strength predicted by these models is unexpectedly finite. To improve the performance of lattice model under compression, the confinement effect has been introduced into the lattice model [17] .

A crucial issue of the lattice method is the bond rupture criterion. So far, many criteria have been developed. In these criteria, when the quantity of bond, i.e., strain energy, bond force or bond strain, exceeds a threshold, the bond breaks [18-20]. Although these bond rupture criteria are straightforward and physically sound, they can lead to the mesh size (or lattice resolution) dependence problems as found in $[15,16,21,22]$. The simulated fracture energy and load strength are strongly affected by the lattice size, however, the crack pattern is free of such an effect. The underlying reason for mesh size dependence is that the fracture energy is not conserved when varying lattice size. With decreasing lattice size, the energy dissipated for crack creation approaches zero, which contradicts the reality. To address this problem, some scholars [17, 23-26] have related the bond stress-strain relationship to the macro fracture energy based on the conception of crack band [27] while others [23, 28] have developed a bond rupture criterion with the consideration of fracture energy. In contrast to the incorporation of fracture energy, it has been reported that the mesh size dependency also can be relieved through consideration of statistical distributions of lattice beam strengths in $[29,30]$.

From another stand point, the incorporation of fracture energy can be considered as the introduction of a characteristic length scale into the bond fracture criterion. In the cohesive finite element method, the cohesive law (a stress-displacement relationship) of an interface can preserve the fracture energy while in the conventional continuum methods the constitutive relation (a stress-strain relationship) is unable to do so. The essential difference between the cohesive law and the constitutive relation lies in the length scale parameter. Just as suggested in [12], a length scale characteristic of the mesh must be introduced into the breaking rule in order to eliminate the mesh size sensitivity, i.e., preserve the fracture energy. This length scale is related to the lattice size.

The common feature of the aforementioned lattice models is that they all have a separate bond rupture criterion. Prior to breaking, the bond is linear or bilinear elastic. Here, we call this kind of lattice method with separate rupture criterion as the extrinsic lattice model. The linear elastic bond cannot capture the hyperelastic property of the material. According to[31, 32], the hyperelasticity governs the dynamic fracture at a critical length scale, and the instability of dynamic fracture. Therefore, the extrinsic lattice model has a critical drawback in dynamic fracture simulation.

In contrast to the extrinsic lattice model, Wang et al [33], Zhang et al [34, 35] introduced the interatomic potentials into the lattice model to characterize the interactions between particles. The 
interatomic potential is hyperelastic, which implicitly contains the bond rupture criterion. No explicit bond rupture criterion is provided in such lattice model. Here, we call this kind of lattice model with an interatomic potential as the intrinsic lattice model. Because the intrinsic lattice model can capture the hyperelastic property of the material at the crack tip, it can simulate the dynamic fracture more accurately [31,32]. However, the intrinsic lattice model cannot preserve the fracture energy with varying lattice size. Hence, it is not reliable for quantitative simulation on fracture problems.

To address the problem of non-conservation of the fracture energy in the intrinsic lattice model and the problem of inability to capture the hyperelastic property of material in the extrinsic lattice model, we propose a hyperelastic-bilinear potential(HBP) for the lattice model. In this potential, the bond is governed by an interatomic potential at the pre-peak stage and by a bilinear cohesive law at the post-peak stage. The deformation limit of the bond is related to the lattice size and the intrinsic fracture energy of the material. By this method, both the hyperelasticity and fracture energy are built into the lattice model, making the fracture simulation more accurate.

\section{Hyperelastic-bilinear potential for lattice model}

\subsection{Analysis on non-conservation of fracture energy in lattice model}

Fig. 1 shows a schematic of a fracture simulated by a lattice bond method. If the bond force or bond strain rupture criterion is adopted, shown as Fig.1b, the simulation results are lattice size-dependent as demonstrated in Fig.1c and Fig.1d. In the coarse lattice scheme (Fig.1c), no bond force ahead of crack tip exceeds its tensile strength $f_{t}$. As result, the crack does not advance. However, in the fine meshing scheme, some bond forces ahead of crack tip will exceed their tensile strength due to the stress intensity. Consequently, the fracture advances. So, the lattice bond method with the bond strain or bond force criterion is sensitive to the lattice size, with the underlying reason that the fracture energy is not conserved, as analyzed in[36].

\subsection{Hyperelastic-bilinear potential}

The bond cohesive law of the intrinsic lattice bond method is shown in Fig.2a. In this cohesive law, the hyperelastic property of the material at pre-peak stage is captured, but it is difficult to incorporate the material's fracture energy. In the extrinsic lattice bond method, the bond cohesive law is shown in Fig.2b. In this cohesive law, the hyperelastic behavior of material at crack tip is missed, but the fracture energy can be embedded by setting the limit strain or defining bond rupture criterion in term of fracture energy and lattice size as introduced in the section of Introduction. To inherit the advantages of the intrinsic and extrinsic bond cohesive law, a combined potential, i.e., the HBP, should be developed for the lattice bond model, whose diagram is shown in Fig.2c. Before bond force reaches its peak, the bond behavior is governed by an interatomic potential while in the post-peak stage, it is governed by a bilinear cohesive law. The conception of the post-peak bilinear cohesive law of bond comes from the bilinear cohesive law of 
cohesive surface developed in[37, 38], in which the total fracture energy $G_{F}$ is partitioned into the initial fracture energy $G_{f}$ and the remaining part.

Let $\Phi$ stand for the HBP, $\Phi=\Phi(\ell)$ with $\ell$ being the bond length. Its first and second derivative with respect to bond length are $\Phi^{\prime}(\ell)=\partial \Phi(\ell) / \partial \ell$ and $\Phi^{\prime \prime}(\ell)=\partial^{2} \Phi(\ell) / \partial \ell^{2}$, respectively. Then, the bond force is derived as:

$$
f=\left\{\begin{array}{ccc}
\Phi^{\prime}(\ell) & \text { if } & \ell \leq \ell_{b} \\
\frac{\left(\ell-\ell_{r}\right) f_{t}}{\ell_{b}-\ell_{r}} & \text { if } & \ell_{b}<\ell \leq \ell_{p} \\
\frac{\left(\ell-\ell_{c}\right) \beta f_{t}}{\ell_{p}-\ell_{c}} & \text { if } & \ell_{p}<\ell \leq \ell_{c} \\
0 & \text { if } & \ell_{c}<\ell
\end{array}\right.
$$

and the bond tangent stiffness is:

$$
k=\left\{\begin{array}{ccc}
\Phi^{\prime \prime}(\ell) & \text { if } & \ell \leq \ell_{b} \\
\frac{f_{t}}{\ell_{b}-\ell_{r}} & \text { if } & \ell_{b}<\ell \leq \ell_{p} \\
\frac{\beta f_{t}}{\ell_{p}-\ell_{c}} & \text { if } & \ell_{p}<\ell \leq \ell_{c} \\
0 & \text { if } & \ell_{c}<\ell
\end{array}\right.
$$

where $\ell_{b}, \ell_{p}, \ell_{r}, \ell_{c}$ are the shape parameters of the HBP.

\subsection{Parameter determination}

The parameter calibration of the classical lattice models is case-dependent. The bond parameters are dependent on the specific topological structure of the unit cell. This makes the calibration of bond parameters especially difficult in 3D or in the cases of topological structure-complicated unit cell. Recently, Zhang[34] has suggested a universal micro-macro parameter relationship for the irregular lattice model, i.e.,

$$
\Phi^{\prime \prime}\left(\ell_{0}\right)=\left\{\begin{array}{lll}
\frac{6 E V}{\Omega \ell_{0}^{2}} & \text { for } & \text { 3D-Case } \\
\frac{3 E V}{\Omega \ell_{0}^{2}} & \text { for } & \text { plane-stress } \\
\frac{16 E V}{5 \Omega \ell_{0}^{2}} & \text { for } & \text { plane-strain }
\end{array}\right.
$$

where $\Phi^{\prime \prime}\left(\ell_{0}\right)$ means the initial stiffness of bond; $E$ is the Young's modulus; $V$ is the volume 
of $3 \mathrm{D}$ unit cell and the area of $2 \mathrm{D}$ unit cell; $\Omega$ is the bond number in a unit cell and $\ell_{0}$ is the bond length in un-deformed state.

The shape parameters of bond include $\ell_{b}, \ell_{r}, \ell_{c}, \ell_{p}$. The $\ell_{b}$, which denotes the bond length corresponding to the peak force of bond, is related to the critical strain $\varepsilon_{t}$ of material at which the uniaxial tensile stress reaches the tensile strength. So, the $\ell_{b}$ has the form:

$$
\ell_{b}=\ell_{0}+\tilde{\varepsilon}_{t} \ell_{0}
$$

where $\tilde{\varepsilon}_{t}$ is the critical bond strain, which is related to the critical strain $\varepsilon_{t}$ and the uniaxial tensile strength of material. To calibrate $\tilde{\varepsilon}_{t}$, we can firstly assign it an initial value $\varepsilon_{t}$ and then revise it by fitting a uniaxial tensile test. The critical bond strain can be determined as $\tilde{\varepsilon}_{t}=\varepsilon_{t} \sigma_{t} / \tilde{\sigma}_{t}$, in which $\sigma_{t}, \tilde{\sigma}_{t}$ is the tested uniaxial and the simulated uniaxial tensile strength.

The rest of shape parameters $\ell_{p}, \ell_{r}, \ell_{c}$ are related to the initial fracture energy $G_{f}$, the total fracture energy $G_{F}$ and the unit cell size. The $\ell_{p}$ is the transition point of two linear segments of bilinear cohesive law. $\ell_{c}$ is the limit bond length over which bond force vanishes.

According to Fig.2, the work of the bond can be partitioned into three parts. They are:

$$
\begin{aligned}
& W_{1}=\Phi\left(\ell_{b}\right)-\Phi\left(\ell_{0}\right) \\
& W_{2}=\frac{1}{2}\left(\ell_{r}-\ell_{b}\right) f_{t} \\
& W_{3}=\frac{1}{2}\left(\ell_{c}-\ell_{r}\right) \beta f_{t}
\end{aligned}
$$

where $W_{1}$ is the work contributed by the hyperelastic potential; $W_{2}, W_{3}$ are the works contributed by the bilinear cohesive law; $f_{t}$ is the maximum bond force, $f_{t}=\Phi^{\prime}\left(\ell_{b}\right)$.

To relate the bond work to fracture energy, a representative unit bond cell is considered. According to[34, 35], a unit bond cell can take any geometric figure and can have any number of bonds. When a unit cell is overstretched, a crack surface is created in it (Fig.3). To quantify the crack surface or the facet area, the characteristic size of the unit cell is firstly defined as:

$$
h=\left\{\begin{array}{lll}
V^{1 / 2} & \text { for } & \text { 2D-Case } \\
V^{1 / 3} & \text { for } & \text { 3D-Case }
\end{array}\right.
$$

The area of the created crack facet can be quantified as: 


$$
S=\gamma h^{2}
$$

where $\gamma$ is a coefficient, relating to the geometry of the unit cell.

The energy dissipated to create a crack facet is offered by the bonds cut by the crack facet. So, according to the definition of fracture energy, we have:

$$
\begin{aligned}
G_{f} & =\frac{N\left(W_{1}+W_{2}\right)}{S} \\
G_{F} & =\frac{N\left(W_{1}+W_{2}+W_{3}\right)}{S}
\end{aligned}
$$

where $N$ is the number of bonds cut by crack facet in a unit cell. The value of $N$ is related to the specific topology of a unit cell. For example, in the triangular unit cell (Fig.3b), $N=2$ while in the tetrahedral unit cell, $N=3$ or $N=4$, depending on the cut mode, shown in Fig.3c.

With Eqs.(5-8) and the geometrical relationship among $\ell_{p}, \ell_{r}$ and $\ell_{c}$, shown in Fig.2c, we derive the shape parameters of $\mathrm{HBP}$ as:

$$
\begin{aligned}
& \ell_{r}=\ell_{b}+\frac{2\left(\theta G_{F} h^{2} \gamma / N-W_{1}\right)}{f_{t}} \\
& \ell_{c}=\ell_{r}+\frac{2(1-\theta) G_{F} h^{2} \gamma / N}{\beta f_{t}} \\
& \ell_{p}=\ell_{r}-\beta\left(\ell_{r}-\ell_{b}\right)
\end{aligned}
$$

where $\theta$ is the fracture energy ratio, $\theta=G_{f} / G_{F} ; \beta$ is the bond force ratio. In Eq.(9), the ratio $\gamma / N$ is related to the geometry of a unit cell. Thus, we can consider $\gamma / N$ as an integrated geometrical parameter to calibrate. For the irregular triangular unit cell, it is calibrated as:

$$
\gamma / N \approx 0.33
$$

While for the irregular tetrahedral unit cell, it is:

$$
\gamma / N \approx 0.15
$$

For the detailed calibration method of $\gamma / N$, see Appendix A.

It is worthy of pointing out that a critical unit cell size is hinted in Eq.(9). That is

$$
h_{\text {critical }}=\frac{W_{1}}{\theta G_{F} \gamma / N}
$$

If the unit cell is larger than its critical size, $\ell_{r}$ will be smaller than $\ell_{b}$. This violates the underlying hypothesis of the HBP. So, the HBP is applied to unit cells which are smaller than the critical size. For the unit cell larger than the critical size, we just use the original hyperelastic 
interatomic potential $\Phi(\ell)$.

\section{Verification}

To examine whether the proposed HBP can preserve the fracture energy, we use it to simulate a three-point-bend test reported in $[39,40]$. The beam used for three-point-bend test is made of micro-concrete (with maximum aggregate size $5 \mathrm{~mm}$ ). Yu et al[40] have carried out a comprehensive simulation on this experiment using different numerical methods. This three-point-bend test reported in $[39,40]$ is representative, and it can be numerically reproduced since the detailed parameters are provided. The beam geometry and the meshing scheme are shown in Fig.4. To exclude the nonlinearity effect from material outside of crack zone, we only treat the triangular elements in the middle of the beam, where crack path runs, using the lattice bond cells while the rest of the elements are treated as linear elastic bulk triangular elements.

The two-parameter hyperelastic potential in[41] is employed:

$$
\Phi(\ell)=-A B \exp \left(-\frac{\ell-\ell_{0}}{B}\right)\left(\ell-\ell_{0}+B\right)
$$

where $B=\ell_{b}-\ell_{0}=\tilde{\varepsilon}_{t} \ell_{0}$. Its first and second derivative are

$$
\Phi^{\prime}(\ell)=A\left(\ell-\ell_{0}\right) \cdot \exp \left(-\left(\ell-\ell_{0}\right) / B\right) \quad \text { and }
$$

$\Phi^{\prime \prime}(\ell)=A \exp \left(-\left(\ell-\ell_{0}\right) / B\right)\left(1-\left(\ell-\ell_{0}\right) / B\right)$, respectively.

The given material parameters in [40] are: the uniaxial tensile strength $\sigma_{t}=3.8 \mathrm{MPa}$; the Young's modulus $E=30.5 \mathrm{GPa}$; the Poisson ratio $v=0.2$; the total fracture energy $G_{F}=62.5 \mathrm{~J} / \mathrm{m}^{2}$ and the material density $\rho=2402 \mathrm{~kg} / \mathrm{m}^{3}$. The critical bond strain is calibrated as $\tilde{\varepsilon}_{t}=0.2765 \times 10^{-3}$ according to the given $E$ and $\sigma_{t}$. The fracture energy ratio is set as $G_{f} / G_{F}=0.3$ and the bond force ratio as $\beta=0.2$. With these parameters, the simulated macro uniaxial tensile stress-strain curve is shown in Fig.5.

The simulated force-displacement curves with different mesh sizes in 2D cases are shown in Fig.6. According to Fig.6, the simulated results by the present method are almost independent of the mesh size, while those obtained from the conventional hyperelastic potential are strongly dependent on the mesh size. The same is true in 3D cases, shown in Fig.7. This demonstrates that the present method can preserve the fracture energy and eliminates the element size effect.

The fracture energy ratio $\theta$ and the bond force ratio $\beta$ have significant influence on the macro mechanical properties of material. As shown in Fig.8, the fracture energy ratio dominates the peak strength while the bond force ratio dominates the post-peak behaviors. 


\section{4 simulation examples}

\subsection{Quasi-static fracture case}

To examine the performance of the present method in quasi-static fracture simulation, we use it to simulate a fracture propagation case reported in [42]. Galvez et al[42] have conducted experiments on the mixed fracture propagation in the notched concrete beams, whose geometry is shown in Fig.9a. In present simulation the triangular unit cells are adopted. The meshing scheme is shown in Fig.9b. The average parameters of concrete (see Table 2 in [42]) are $E=38.4 \mathrm{GPa}$; $G_{F}=68.8 \mathrm{~J} / \mathrm{m}^{2} ; \sigma_{c}=56.8 \mathrm{MPa} ; \sigma_{t}=3.0 \mathrm{MPa}$. According to these given parameters, the parameters adopted in simulation are: $E=38.4 \mathrm{GPa} ; \quad \tilde{\varepsilon}_{t}=0.1829 \times 10^{-3} ; G_{F}=68.2 \mathrm{~J} / \mathrm{m}^{2}$; $\theta=0.3$ and $\beta=0.2$. The simulated results are shown in Fig.10. From Fig.10 it is seen that the simulated load-displacement curves reasonably agree with the experimental results by [42]. The simulated peak values of load are close to the tested ones.

\subsection{Dynamic fracture case}

To examine the performance of the present method in dynamic fracture simulation, we use it to simulate a compact concrete beam with an offset crack as reported in [43, 44]. John and Shah[44] conducted an experimental study to investigate the mixed mode fracture of concrete subjected to an impact load. The experimental specimens are concrete beams with notches at different locations along the span of the beams. The setup of the specimen is shown in Fig.11a. Belytschko and Tabbara[43] have simulated this experiment using the element-free Galerkin method. To facilitate the comparison of analyses, we used the same material parameters and loading scheme as in [43] in simulating the experiment. The discretization scheme with tetrahedral unit cell is shown in Fig.11b. The total node number is 37,462 and the total unit cell number is 210,653 .

The beam is subjected to a velocity [43]:

$$
V_{0}(t)=\left\{\begin{array}{ccc}
V_{1} t / t_{1} & \text { for } & t \leq t_{1} \\
V_{1} & \text { for } & t>t_{1}
\end{array}\right.
$$

at the upper edge shown as Fig.11a, where $t_{1}=1.96 \times 10^{-4} \mathrm{~s}$ and $V_{1}=0.065 \mathrm{~m} / \mathrm{s}$.

The parameters given in [43] are: $E=31.37 \mathrm{GPa} ; \quad v=0.20 ; \rho=2400 \mathrm{~kg} / \mathrm{m}^{3}$; $K_{I}^{c}=0.8 \mathrm{MPa} \sqrt{\mathrm{m}}$. According to these parameters and the uniaxial tensile strength $\sigma_{t}=2.99 \mathrm{MPa}$ as suggested in [35], the critical bond strain is calibrated as $\tilde{\varepsilon}_{t}=0.212 \times 10^{-3}$.

Take the total fracture energy $G_{F}=62.5 \mathrm{~J} / \mathrm{m}^{2}$. Set the bond force ratio $\beta=0.2$ and fracture 
energy ratio $\theta=0.3$. With $\theta=0.3$, the initial fracture energy $G_{f}=0.3 G_{F} \approx 19.0 \mathrm{~J} / \mathrm{m}^{2}$ which is approximately equal to the fracture energy $\left(K_{I}^{c}\right)^{2} / E \approx 20.4 \mathrm{~J} / \mathrm{m}^{2}$ evaluated by the $K_{I}^{c}$ and $E$ given above. The explicit integration scheme is adopted with the time interval $\Delta t=0.05 \mu \mathrm{s}$. With these parameters, the simulated macro uniaxial stress-strain curve is shown in Fig.12.

To examine the effect of fracture energy conservation on dynamic fracture behavior, we simulate this impact beam in two cases:

Case I: using the present HBP(Eq.(1));

Case II: using the conventional hyperelastic potential $\Phi(\ell)$ (Eq.(11)) .

The simulated fracture profiles and morphologies are shown in Fig.13. From Fig.13 it is seen that in the Case I where fracture energy is conserved, the inclination of fracture with respect to $\mathrm{x}$-axis is about $65.3^{\circ}$, which falls into the fracture inclination range $61.2^{\circ} \sim 77.4^{\circ}$ reported in[43]. The simulated fracture inclination in[43] is $68.4^{\circ}$, close to the value $65.3^{\circ}$ simulated in Case I. However, the simulated fracture inclination in Case II is about $51.3^{\circ}$ (Fig.13b), which falls out of the reported inclination range and deviates from the simulated value $68.4^{\circ}$ significantly. Fig.14 shows the simulated results by [43] and the test results by[44].

Fig. 15 shows the simulated crack speed in two cases, which clearly demonstrates that the fracture energy has a strong influence on the crack speed. The crack speed with fracture energy conservation is much slower than that without fracture energy conservation. According to Fig.14 a,b,c, we can evaluate the extended crack lengths $L_{1} \approx 3.2 \mathrm{~mm}$ at $t_{1}=720.3 \mu \mathrm{s}$; $L_{2} \approx 18.4 \mathrm{~mm}$ at $t_{2}=842.8 \mu \mathrm{s} ; L_{3} \approx 33.8 \mathrm{~mm}$ at $t_{3}=980.0 \mu \mathrm{s}$. The crack propagation initiation time predicted in [43] is $t_{0}=686.0 \mu \mathrm{s}$. Therefore, we can evaluate the average crack speeds in the three stages are respectively $v_{1} \approx 93.0 \mathrm{~m} / \mathrm{s} ; v_{2} \approx 124.0 \mathrm{~m} / \mathrm{s} ; v_{3} \approx 112.0 \mathrm{~m} / \mathrm{s}$, which are very close to the simulated values in Case I, indicated in Fig.15b. However, the crack speed without fracture energy conservation is more than two times this value. From Fig.15a it is also found that the time to crack propagation is about $450 \mu s$ in Case I while $360 \mu s$ in Case II. The fracture energy conservation delays the time needed for the onset of fracture propagation.

The quantitative comparison suggests that the HBP can accurately simulate fracture behavior. 
However, the present method still has some limitations. After material undergoes a large deformation, some irreversible processes (e.g., damage, plasticity) occur. The HBP cannot capture these irreversible processes. When unloaded, the deformation governed by this HBP will be completely recovered. So, the HBP is not applicable to the cyclic loading conditions. But it can represent the resultant response of the damage and plasticity. Due to the damage and plastic deformation, the stiffness of material reduces. The pre-peak hyperelastic behavior of bond can reflect the weakness of the material under monotonic loading conditions. So, the HBP in its current form is only applicable to monotonic loading conditions.

Under dynamic loading conditions, the viscosity effect is not negligible, and can be even significant. The HBP is hyperelastic. Therefore, it cannot capture the viscosity effect. The rate dependency effects demonstrated in the simulation using HBP are rooted in the inertia effect.

\section{Conclusions}

The proposed HBP can preserve the fracture energy in the lattice model. By this HBP, the mesh-size sensitivity can be almost eliminated in fracture simulation. The effect of fracture energy conservation is significant in dynamic fracture simulation. It lowers the crack speed, bringing it closer to the real crack speed. The simulation results by the HBP are much more accurate. But the $\mathrm{HBP}$ in its current form is only applicable to the monotonic loading conditions.

\section{Acknowledgement}

The present work is supported by the National Natural Science Foundation of China (No. 11172172) and the National Basic Research Program of China (Grant No.2011CB013505), which are gratefully acknowledged.

\section{Appendix A}

To calibrate the ratio $\gamma / N$ for the irregular triangular unit cells, we discretized a square with unstructured meshing scheme, shown in Fig.A1, and then used a straight line, which is taken as crack, to cut through this square. Consequently, many unit cells are cut by this segment. Assume that $\gamma$ is constant. The computed crack area is computed as:

$$
S=\sum_{i=1}^{n} \gamma V_{i}^{1 / 2}=\gamma \sum_{i=1}^{n} V_{i}^{1 / 2}
$$

where $n$ is the total number of triangular unit cells cut by 'crack'.

Let $S_{\text {exact }}$ denote the exact crack area. The $\gamma$ should take such value that can make

$$
S=S_{\text {exact }} \Rightarrow \gamma=S_{\text {exact }} / \sum_{i=1}^{n} V_{i}^{1 / 2}
$$

For the 2D case, the exact crack area is equal to the length of the straight line segment intersected by the square, i.e. $S_{\text {exact }}=L$. 
After many times of changing the meshing scheme and the orientation of the straight line, we found that the values of $\gamma$ in different cases always fluctuate around about 0.66 . For a triangular unit cell, the number of bond cut by a crack is $N=2$ (Fig.3b). So, we suggested $\gamma / N \approx 0.33$.

For the 3D cases with irregular tetrahedral unit cells, we take the same method to calibrate the geometrical ratio. First, we mesh a cube with unstructured tetrahedral unit cells and use a plane to cut through this cube. Then, the computed crack area is

$$
S=\sum_{i=1}^{n} \gamma V_{i}^{1 / 3}=\gamma \sum_{i=1}^{n} V_{i}^{1 / 3}
$$

The exact 'crack' area is the intersection area of the plane and the cube, which can be calculated by the plane function and the cube dimensions. The value $\gamma$ is identified as

$$
\gamma=S_{\text {exact }} / \sum_{i=1}^{n} V_{i}^{1 / 3}
$$

As calibrated in 2D cases, we found that the values of $\gamma$ in different cases always fluctuate around a certain value. For a tetrahedral unit cell, the number of bond cut by a crack is $N=3$ or $N=4$ (Fig.3c). Considering the ratio of the population of unit cells with 4 bonds cut to that with 3 bonds cut, we suggested $\gamma / N \approx 0.15$ for the tetrahedral unit cell cases.

\section{References}

[1] Hahn M, Wallmersperger T, Kroplin BH. Discrete element representation of continua: proof of concept and determination of the material parameters. Comput Mater Sci 2010; 50: 391-402.

[2] Hrennikoff A. Solution of problems of elasticity by the framework method. J Appl Mech 1941; A169-A175.

[3] Carta G, Jones IS, Brun M, Movchan NV, Movchan AB. Crack propagation induced by thermal shocks in structured media. Int J Solids Struct 2013; 50: 2725-2736.

[4] Elias J, Stang H. Lattice modeling of aggregate interlocking in concrete. Int J Fract 2012; 175: $1-11$.

[5] Heizler S, Kessler DA, Elbaz YS. Microbranching in mode-I fracture in a randomly perturbed lattice. Phys Rev E 2013; 88: 022401.

[6] Leite JPB, Slowik V, Mihashi H. Computer simulation of fracture processes of concrete using mesolevel models of lattice structures. Cement Concrete Res 2004; 34: 1025-1033.

[7] Lilliu G, Van Mier JGM. 3D lattice type of fracture model for concrete. Eng Fract Mech 2003; 70: 927-941.

[8] Buxton GA, Care CM, Cleaver DJ. A lattice spring model of heterogeneous materials with plasticity. Modelling Simul Mater Sci Eng 2001; 9: 485-497.

[9] Chen H, Lin E, Liu Y. A novel Volume-compensated Particle method for 2D elasticity and plasticity analysis. Int J Solids Struct 2014; 51: 1819-1833.

[10] Kale S, Ostoja-Starzewski M. Elastic-Plastic-Brittle transitions and avalanches in disordered media. Phys Rev Lett 2014; 112: 045503. 
[11] Picallo CB, Lopez JM, Zapperi S, Alava MJ. From brittle to ductile fracture in disordered materials. Phys Rev Lett 2010; 105: 155502.

[12] Jagota A, Bennison SJ. Element breaking rules in computational models for brittle fracture. Modelling. Simul. Mater Sci Eng 1995; 3: 485-501.

[13] Jirásek M, Bazant Z. Macroscopic fracture characteristics of random particle systems. Int J Fract 1995; 69: 201-228.

[14] Monette L, Anderson MP. Elastic and fracture properties of the two-dimensional triangular and square lattice. Modell Simul Mater Sci Eng 1994; 2: 53-66.

[15] Schlangen E. Computational aspects of fracture simulations with lattice models Fracture Mechanics of concrete structures ( Proc., FraMCoS-2, Zurich) FH Wittmann, ed., Aedificatio, Freiburg, Germany, 1995: 913-928.

[16] Schlangen E, Garboczi EJ. Fracture simulations of concrete using lattice models: computational aspects. Eng Fract Mech 1997; 57: 319-332.

[17] Cusatis G, Bazant Z, Cedolin L. Confinement-Shear lattice model for concrete damage in tension and compression: I. Theory. J Eng Mech 2003; 129: 1439-1448.

[18] Ostoja-Starzewski M, Sheng P, Alzebdeh K. Spring network models in elasticity and fracture of composites and polycrystals. Comput Mater Sci 1996; 7: 82-93.

[19] Wang G, Al-Ostaz A, Cheng AHD, Mantena PR. Hybrid lattice particle modeling: Theoretical considerations for a 2D elastic spring network for dynamic fracture simulations. Comput Mater Sci 2009; 44: 1126-1134.

[20] Zhao SF, Zhao GF. Implementation of a high order lattice spring model for elasticity. Int J Solids Struct 2012; 49: 2568-2581.

[21] Man HK, Van Mier JGM. Size effect on strength and fracture energy for numerical concrete with realistic aggregate shapes. Int J Fract 2008; 154: 61-72.

[22] Mungule M, Raghuprasad BK. Meso-scale studies in fracture of concrete: A numerical simulation. Comput Struct 2011; 89: 12-920.

[23] Arslan A, Ince R, Karihaloo B. Improved lattice model for concrete fracture. J Eng Mech 2002; 128:57-65.

[24] Berton S, Bolander JE. Crack band model of fracture in irregular lattices. Comput Methods Appl Mech Eng 2006; 195: 7172-7181.

[25] Cusatis G, Bazant ZP, Cedolin L. Confinement-shear lattice CSL model for fracture propagation in concrete. Comput Methods Appl Mech Eng 2006; 195: 7154-7171.

[26] Yip M, Li Z, Liao B, Bolander JE. Irregular lattice models of fracture of multiphase particulate materials. Int J Fract 2006; 140: 113-124.

[27] Bazant ZP, Oh BH. Crack band theory for fracture of concrete. Mater Struct 1983; 16: 155-176

[28] Kosteski L, DAmbra RB, Iturrioz I. Crack propagation in elastic solids using the truss-like discrete element method. Int J Fract 2012; 174: 139-161.

[29] Grassl P, Jirasek M. Meso-scale approach to modelling the fracture process zone of concrete subjected to uniaxial tension. Int J Solids Struct 2010; 47: 957-968.

[30] Joseph C, Jefferson AD. Stochastic regularisation of lattice modelling for the failure of quasi-brittle materials. Proceedings of the 6th International Conference on Fracture Mechanics of Concrete and Concrete Structures - Fracture Mechanics of Concrete and Concrete Structures, 2007. 1: 445-452. 
[31] Buehler MJ, Abraham FF, Gao HJ. Hyperelasticity governs dynamic fracture at a critical length scale. Nature 2003; 426: 141-146.

[32] Buehler MJ, Gao HJ. Dynamical fracture instabilities due to local hyperelasticity at crack tips. Nature 2006; 439: 307-310.

[33] Wang G, Al-Ostaz A, Cheng AHD, Mantena PR. Hybrid lattice particle modeling of wave propagation induced fracture of solids. Comput Methods Appl Mech Eng 2009; 199: 197-209.

[34] Zhang Z. Discretized virtual internal bond model for nonlinear elasticity. Int J Solids Struct 2013; 50: 3618-3625.

[35] Zhang Z, Chen Y. Modeling nonlinear elastic solid with correlated lattice bond cell for dynamic fracture simulation. Comput Methods Appl Mech Eng 2014; 279: 325-347.

[36] Zhang Z, Huang K. A simple J-integral governed bilinear constitutive relation for simulating fracture propagation in quasi-brittle material. Int J Rock Meck Min 2011; 48: 294-304.

[37] Bazant ZP, Yu Q. Size-effect testing of cohesive fracture parameters and nonuniqueness of work-of-fracture method. J Eng Mech 2011; 137: 580-588.

[38] Evangelista Jr F, Roesler JR, Proenca S. Three-dimensional cohesive zone model for fracture of cementitious materials based on the thermodynamics of irreversible processes. Eng Fract Mech 2013; 97: 261-280.

[39] Ruiz G. Influencia del Tamano y de la Adherencia en la Armadura minima de Vigas en Flexion. Grupo Espanol del Hormigon, ISBN:84-89670-08-0, Madrid, Spain,, 1998.

[40] Yu RC, Ruiz G, Chaves EWV. A comparative study between discrete and continuum models to simulate concrete fracture. Eng Fract Mech 2008; 75: 117-127.

[41] Gao H, Klein PA. Numerical simulation of crack growth in an isotropic solid with randomized internal cohesive bonds. J Mech Phys Solids 1998; 46: 187-218..

[42] Galvez JC, Elices M, Guinea GV, Planas J. Mixed mode fracture of concrete under proportional and nonproportional loading. Int J Fract 1998; 94: 267-284.

[43] Belytschko T, Tabbara M. Dynamic fracture using element-free Galerkin Methods. Int J Numer Methods Engng 1996; 39: 923-938.

[44] John R, Shah SP. Mixed-mode fracture of concrete subjected to impact loading. J Struct Eng 1990; 116: 585-602. 


\section{Figure captions}

Fig.1 Illustration of fracture simulation using lattice bond method and its lattice size dependence (a) a configuration of lattice model to simulate a fracture; (b) elastic-brittle cohesive law of bond; (c) bond force distribution ahead of crack tip in the coarse lattice scheme; (d) bond force distribution ahead of crack tip in the fine lattice scheme (LB denotes the linear elastic bond while EBLB the elastic-brittle bond).

Fig.2 Diagram of the bond cohesive law (a) the interatomic potential; (b) the bilinear cohesive law; (c) the hyperelastic-bilinear potential $\left(G_{f}\right.$ means the initial fracture energy and $G_{F}$ the total fracture energy).

Fig.3 Crack facet created due to an overstretch in (a) the general unit bond cell; (b) the triangular unit cell; (c) the tetrahedral unit cell.

Fig.4 Three-point-bend test specimen and the meshing scheme used (a) Geometry of specimen with a half-notch, where the sample width is $D=0.075 \mathrm{~m}$ and the sample thickness is $0.05 \mathrm{~m}$. The dimensions of specimen are from [40]; (b) 2D meshing scheme with triangular unit cells; (c) 3D meshing scheme with tetrahedral unit cells.

Fig.5 Simulated uniaxial tensile stress-strain curves with calibrated parameters for the three-point-bend test.

Fig.6 Comparison of the simulated (2D) force-displacement curves with different mesh sizes with experimental results from [40]. (a) using the hyperelastic-bilinear potential; (b) using the hyperelastic potential.

Fig.7 Comparing the simulated (3D) force-displacement curves from different mesh sizes with the experimental results with (a) using the hyperelastic-bilinear potential; (b) using the hyperelastic potential.

Fig.8 Influence on the macro mechanical response of (a) fracture energy ratio and (b) bond force ratio.

Fig.9 Simulation set-ups for mixed mode fracture propagation. (a) Geometry of specimen; (b) Meshing scheme. Sample $D 1: \alpha=1.133, D=0.075 \mathrm{~m}$; Sample $D 2: \alpha=1, D=0.15 \mathrm{~m}$; Sample $D 3: \alpha=1, D=0.3 \mathrm{~m}$ (Specimen dimensions are from[42]).

Fig.10 Simulated results of quasi-static mixed fracture propagation (a) Load P versus displacement of point B of different sample sizes; (b) Deformed mesh scheme (Sample D2 whose node displacements are magnified 300 times). The experiment data are from [42].

Fig.11 Notched beam subjected to impact loading (a) Beam dimensions (mm) and boundary conditions (thickness $\mathrm{B}=25.4 \mathrm{~mm}$ ); (b) Illustration of the meshing scheme.

Fig.12 Simulated uniaxial tensile stress-strain curve with calibrated parameters for the impact beam.

Fig.13 Simulated fracture profiles and morphologies (a) Using the hyperelastic-bilinear bond potential; (b) Using the conventional hyperelastic bond potential (in each subfigure, the upper one is the nodes in the last configuration where the displacements are magnified 350 times; the middle one is the extended fracture profile; the bottom one is the extended crack 
surface).

Fig.14 The simulated crack path in[43](Figures 11-13 in [43]) for John's [44] experiments at (a) $t=720.3 \mu \mathrm{s}$; (b) $t=842.8 \mu \mathrm{s}$; (c) $t=980.0 \mu \mathrm{s}$; (d) fracture profile; (e) crack paths emanating from the initial vertical notch reported in [44].

Fig.15 Simulated crack speed (a) Crack speed versus time; (b) Crack speed versus fracture length. (the crack speed is averaged over the time interval5.0 $\mu \mathrm{s}$ ).

Fig.A1 A straight line cut through a square composed of irregular triangular unit cells. 

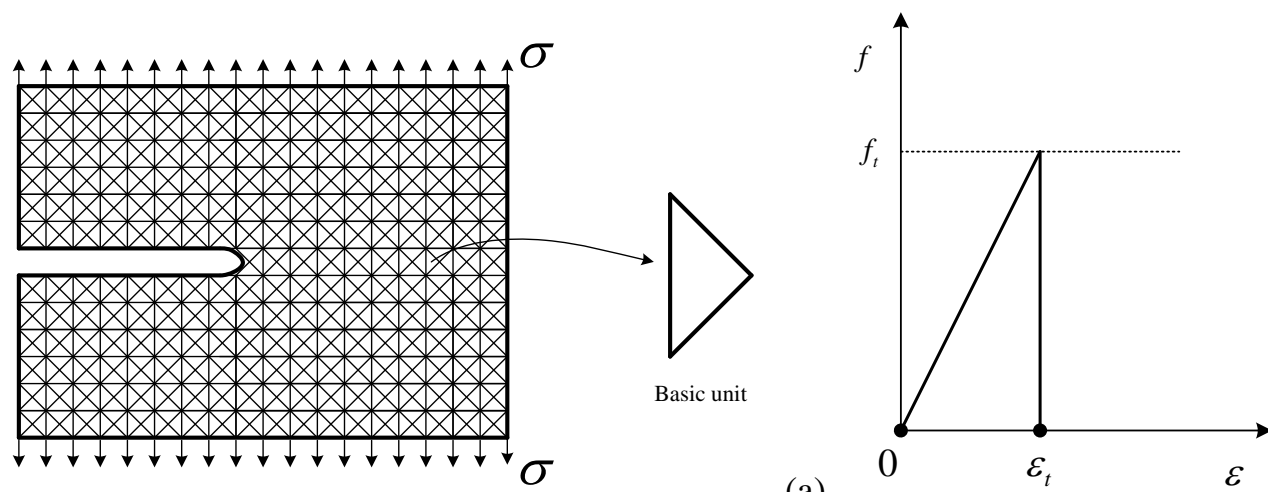

(a)

(b)
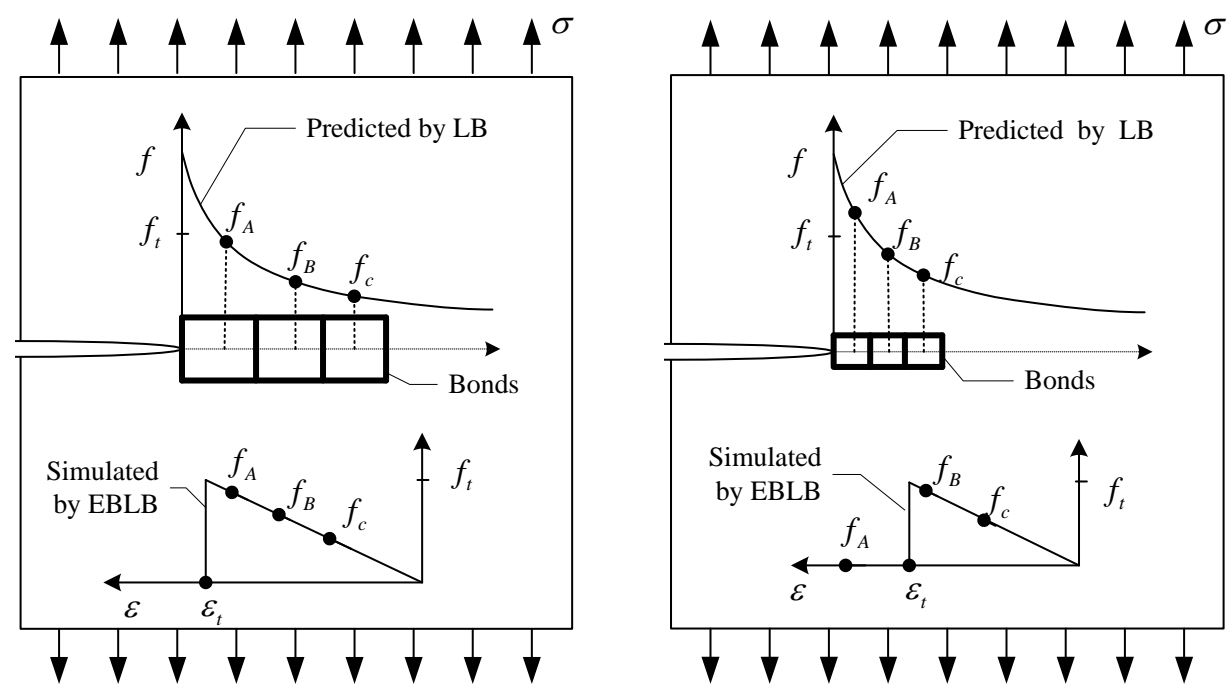

(c)

(d)

Fig.1 Illustration of fracture simulation using lattice bond method and its lattice size dependence (a) a configuration of lattice model to simulate a fracture; (b) elastic-brittle cohesive law of bond;

(c) bond force distribution ahead of crack tip in the coarse lattice scheme; (d) bond force distribution ahead of crack tip in the fine lattice scheme (LB denotes the linear elastic bond while EBLB the elastic-brittle bond).
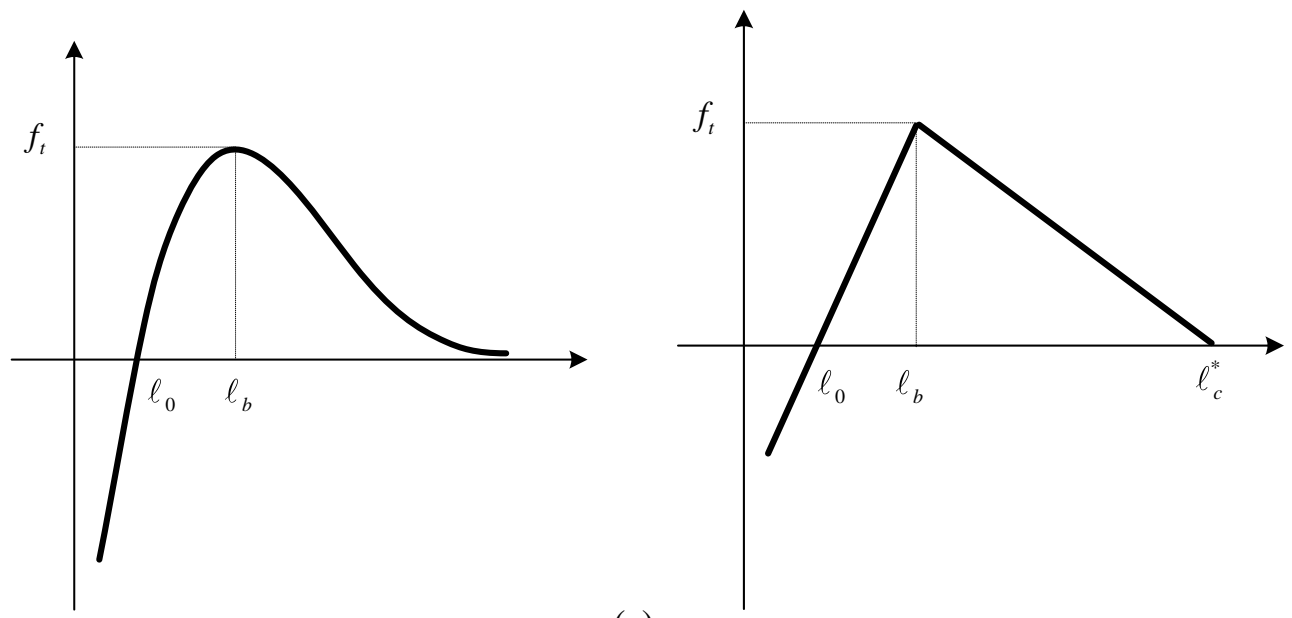

(a) 


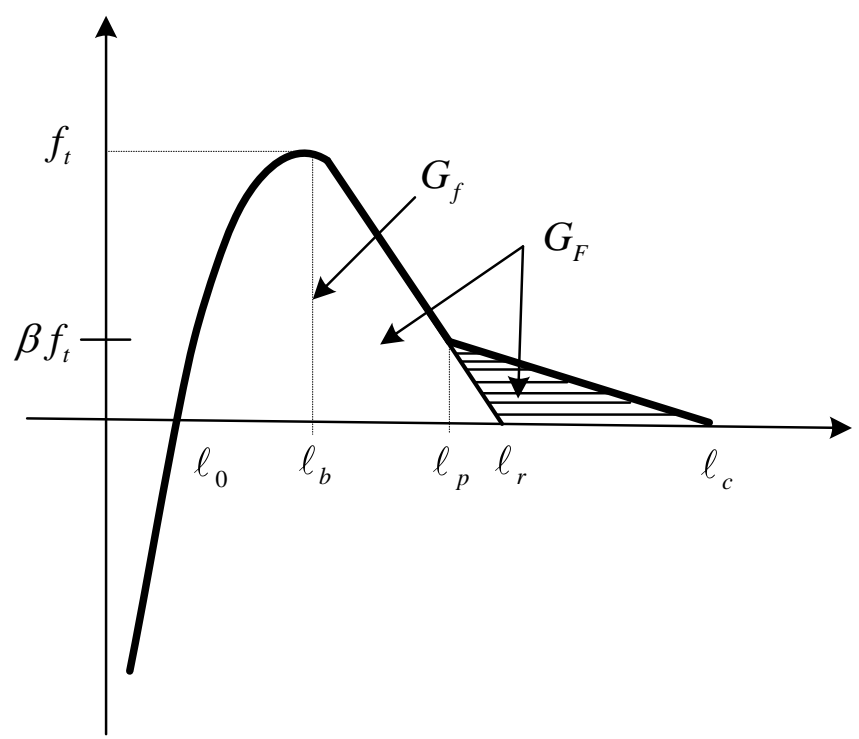

(c)

Fig.2 Diagram of the bond cohesive law (a) the interatomic potential; (b) the bilinear cohesive law; (c) the hyperelastic-bilinear potential $\left(G_{f}\right.$ means the initial fracture energy and $G_{F}$ the total fracture energy).
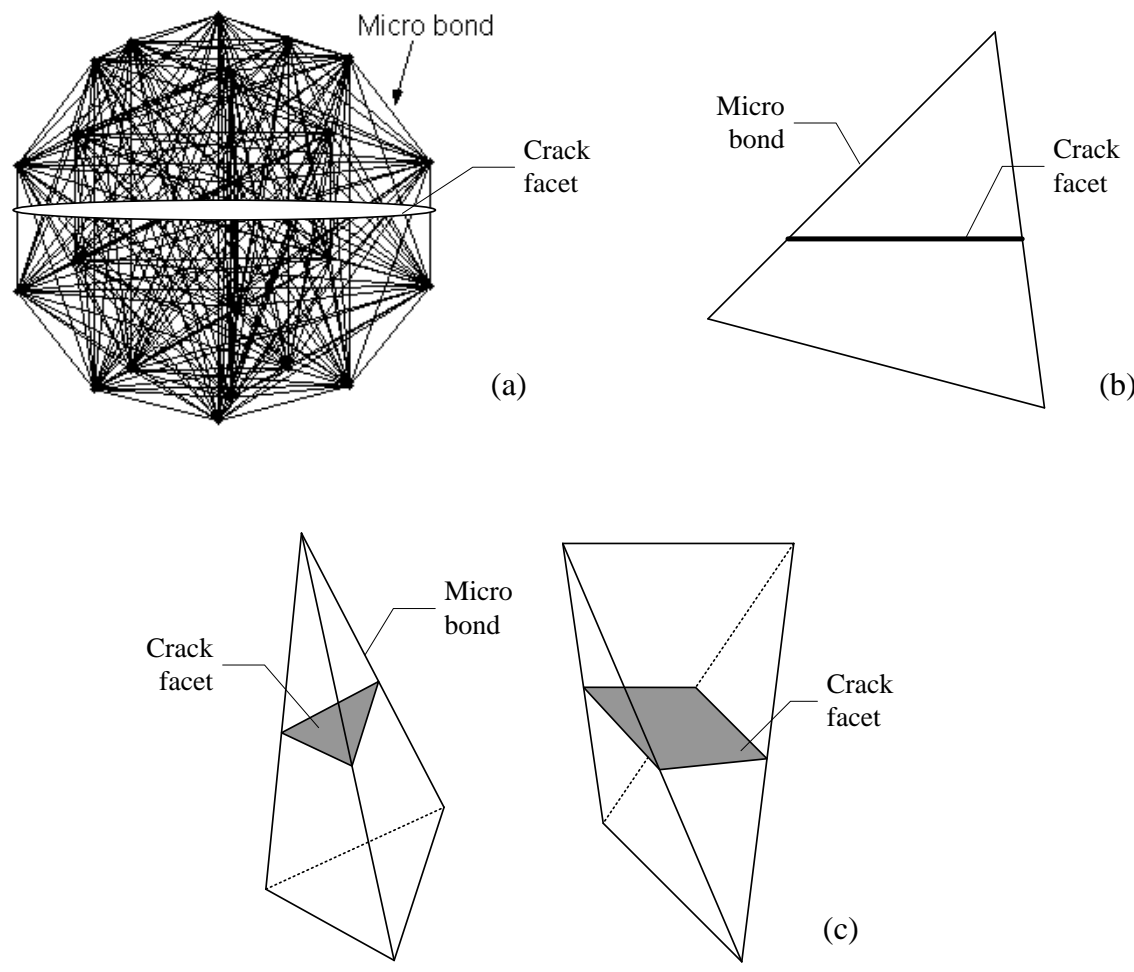

Fig.3 Crack facet created due to an overstretch in (a) the general unit bond cell; (b) the triangular unit cell; (c) the tetrahedral unit cell. 

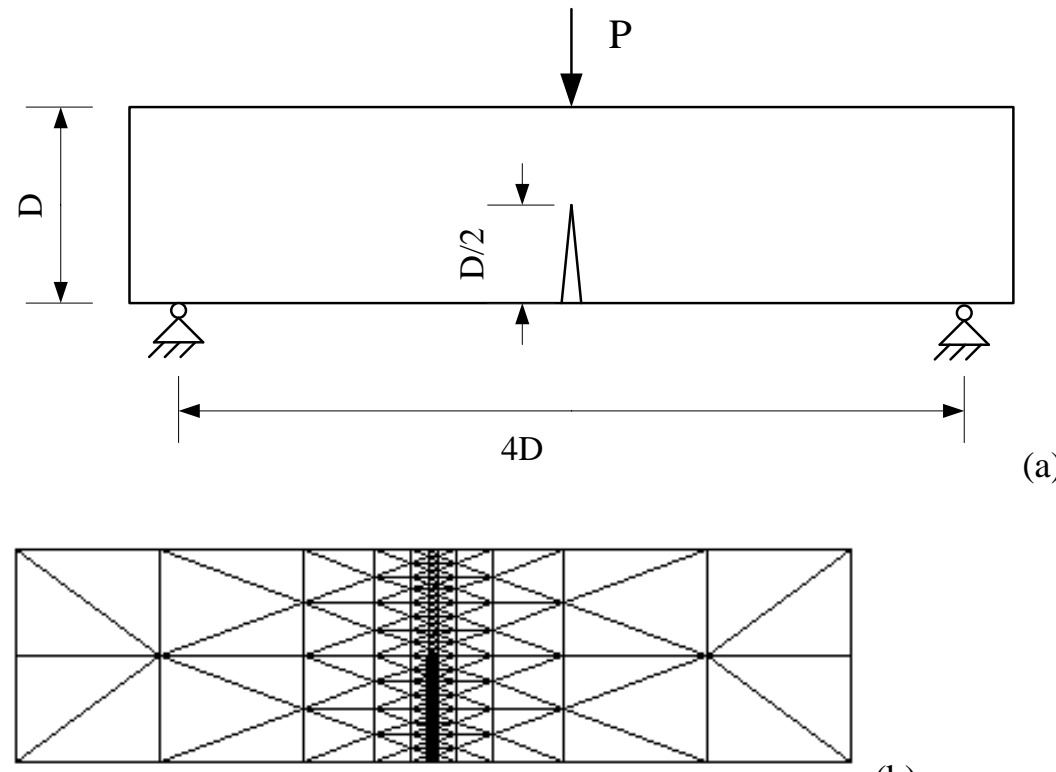

(b)

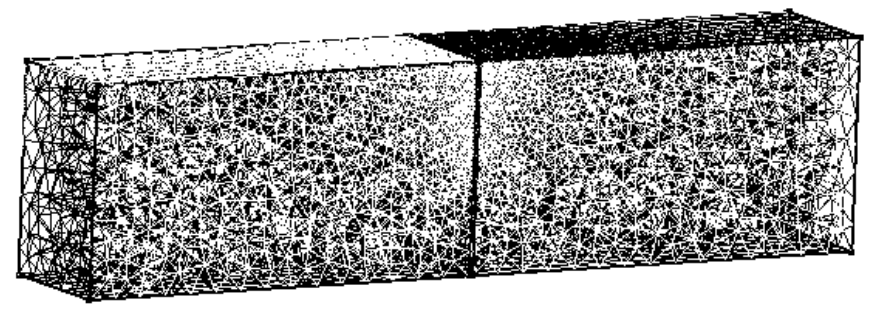

(c)

Fig.4 Three-point-bend test specimen and the meshing scheme used (a) Geometry of specimen with a half-notch, where the sample width is $D=0.075 \mathrm{~m}$ and the sample thickness is $0.05 \mathrm{~m}$. The dimensions of specimen are from [40]; (b) 2D meshing scheme with triangular unit cells; (c) 3D meshing scheme with tetrahedral unit cells.

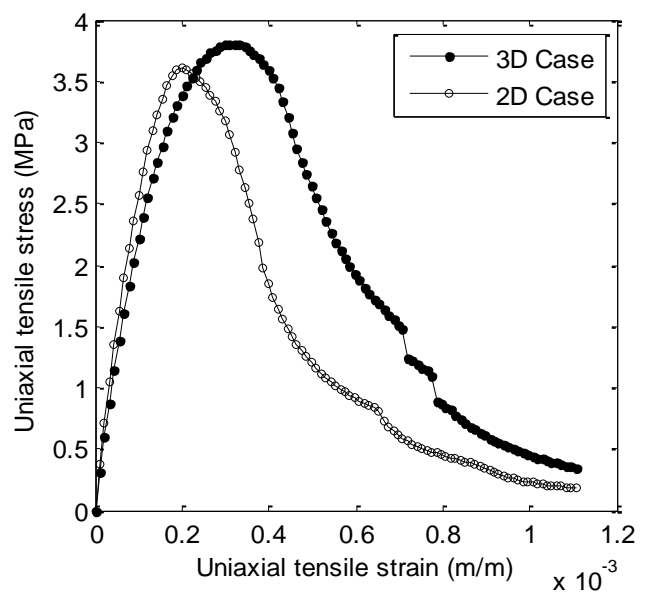

Fig.5 Simulated uniaxial tensile stress-strain curves with calibrated parameters for the three-point-bend test. 

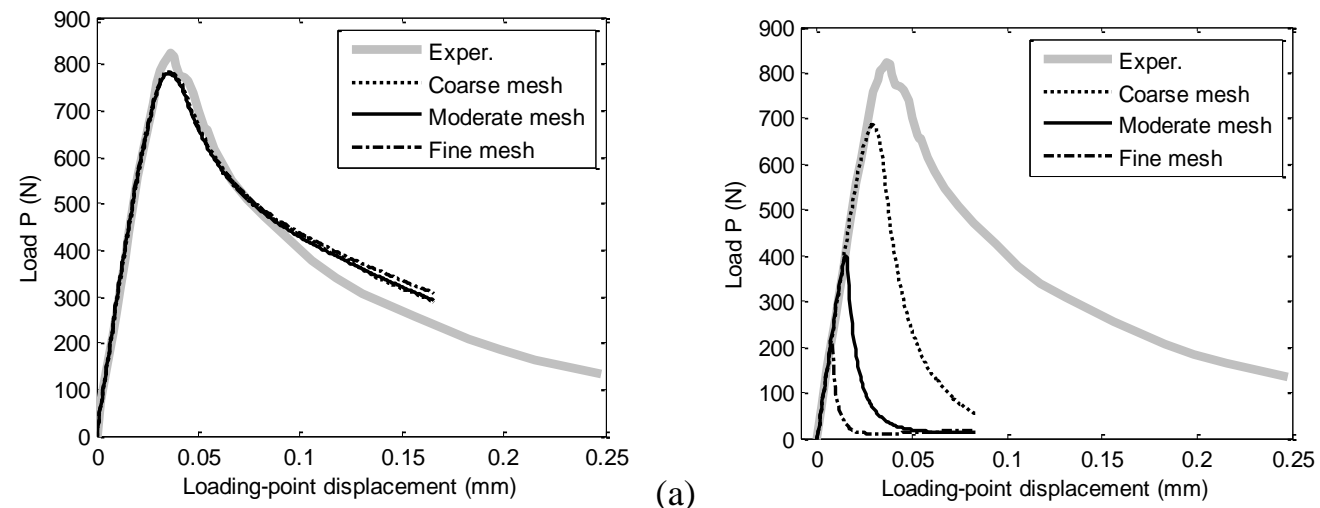

(b)

Fig.6 Comparison of the simulated (2D) force-displacement curves with different mesh sizes with experimental results from [40]. (a) using the hyperelastic-bilinear potential; (b) using the hyperelastic potential.
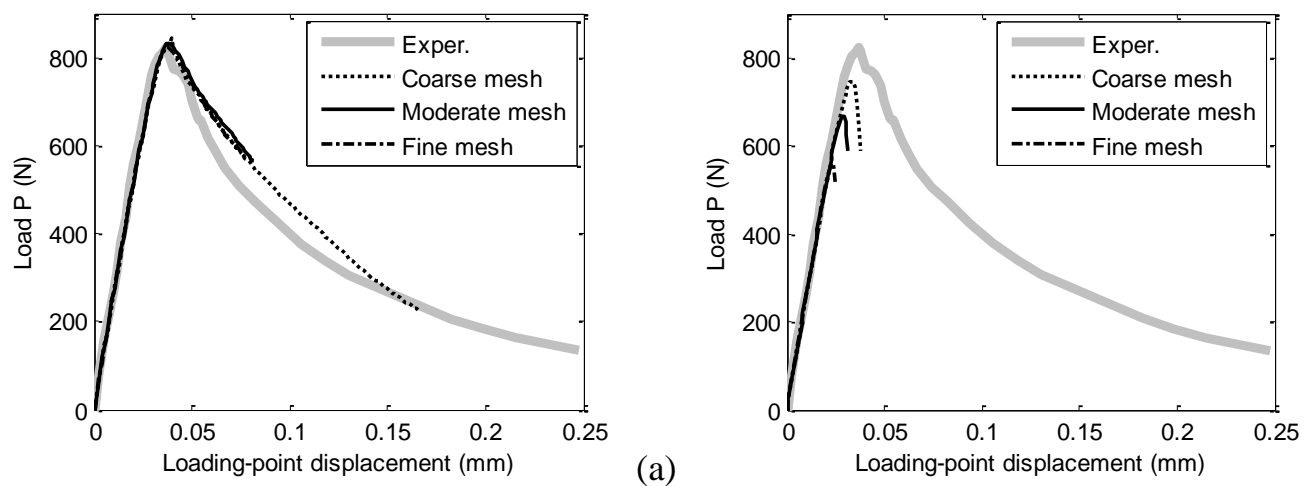

(b)

Fig.7 Comparing the simulated (3D) force-displacement curves from different mesh sizes with the experimental results with (a) using the hyperelastic-bilinear potential; (b) using the hyperelastic potential.
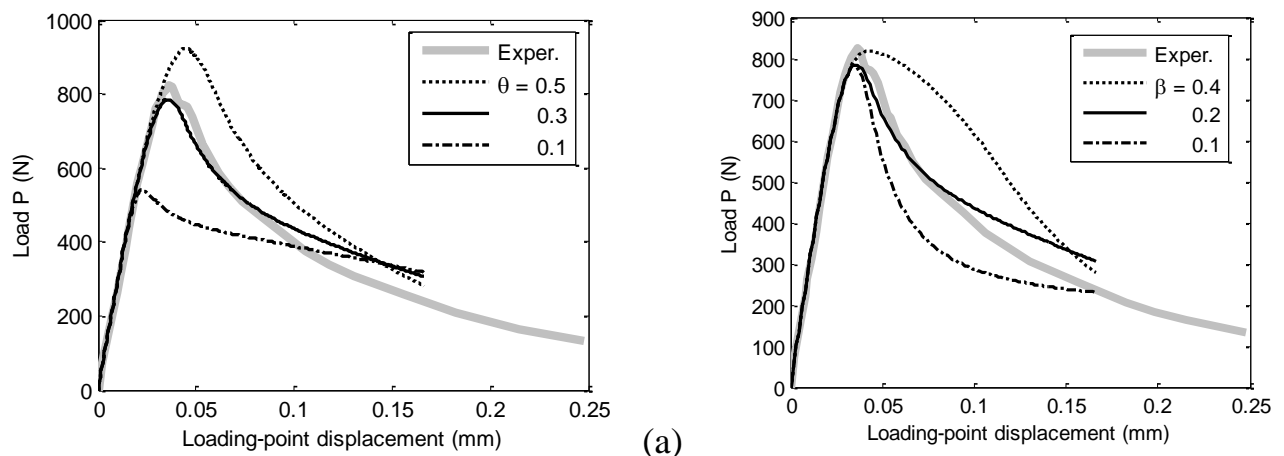

(a)

Fig.8 Influence on the macro mechanical response of (a) fracture energy ratio and (b) bond force ratio. 

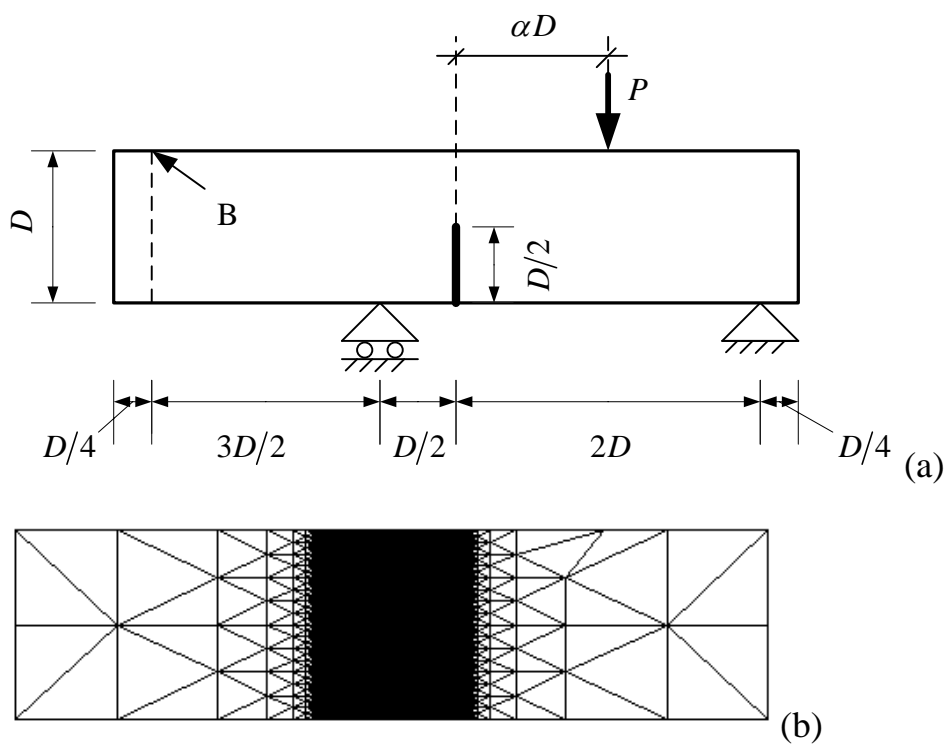

Fig.9 Simulation set-ups for mixed mode fracture propagation. (a) Geometry of specimen; (b) Meshing scheme. Sample $D 1: \alpha=1.133, D=0.075 \mathrm{~m}$; Sample $D 2: \alpha=1, D=0.15 \mathrm{~m}$; Sample $D 3: \alpha=1, D=0.3 \mathrm{~m}$ (Specimen dimensions are from[42]).

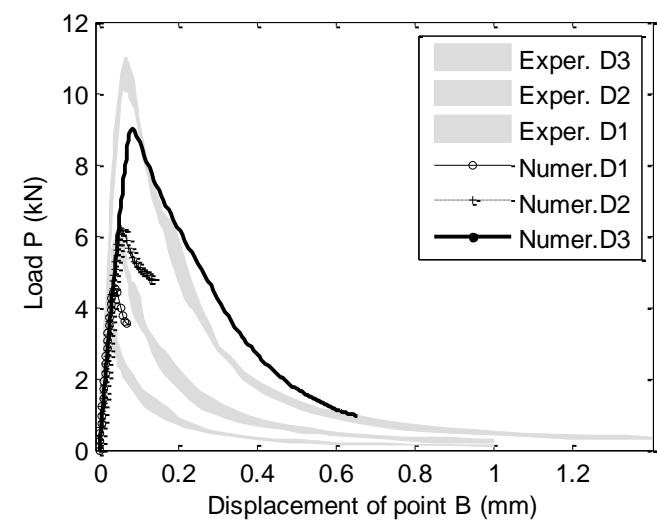

(a)

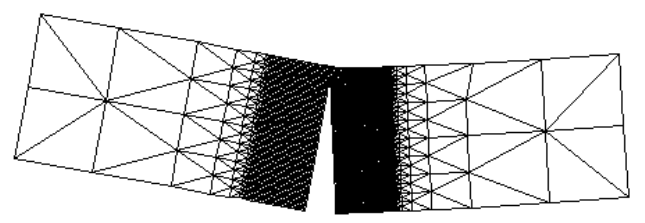

(b)

Fig.10 Simulated results of quasi-static mixed fracture propagation (a) Load P versus displacement of point B of different sample sizes; (b) Deformed mesh scheme (Sample D2 whose node displacements are magnified 300 times). The experiment data are from [42]. 


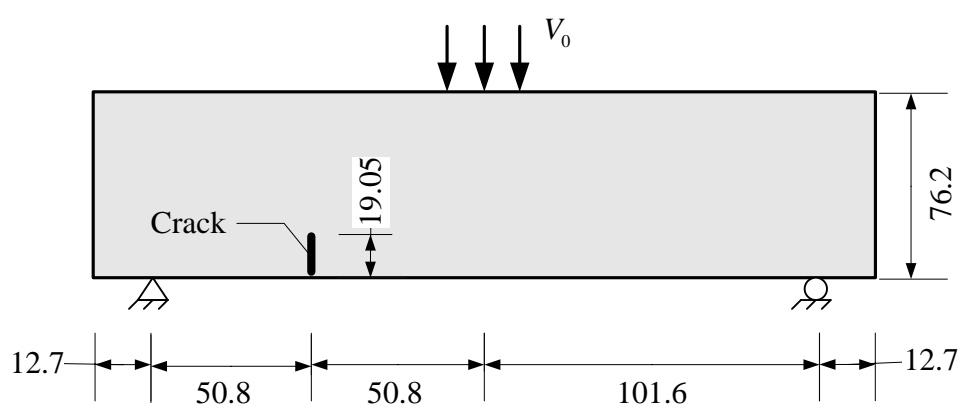

(a)

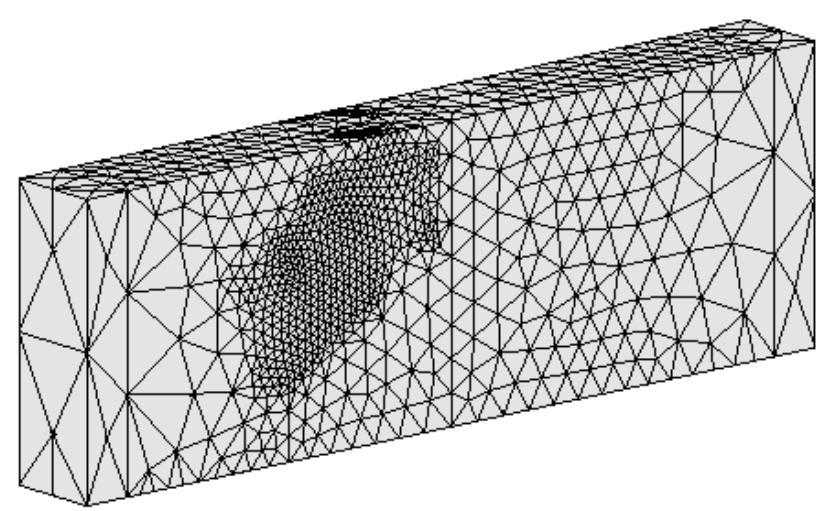

(b)

Fig.11 Notched beam subjected to impact loading (a) Beam dimensions (mm) and boundary conditions (thickness $B=25.4 \mathrm{~mm}$ ); (b) Illustration of the meshing scheme.

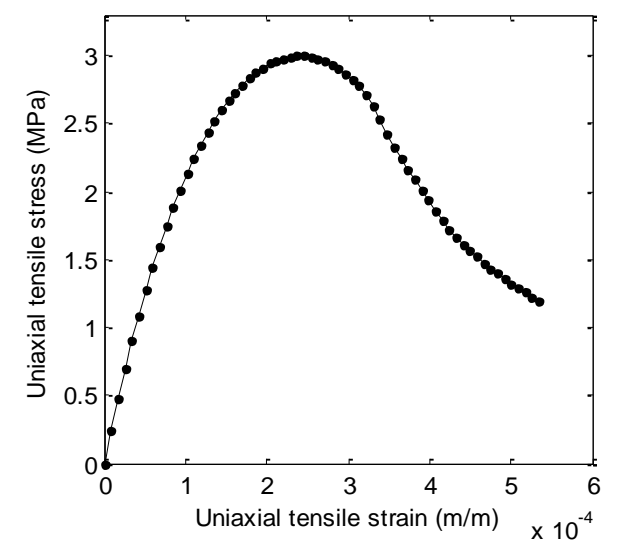

Fig.12 Simulated uniaxial tensile stress-strain curve with calibrated parameters for the impact beam. 

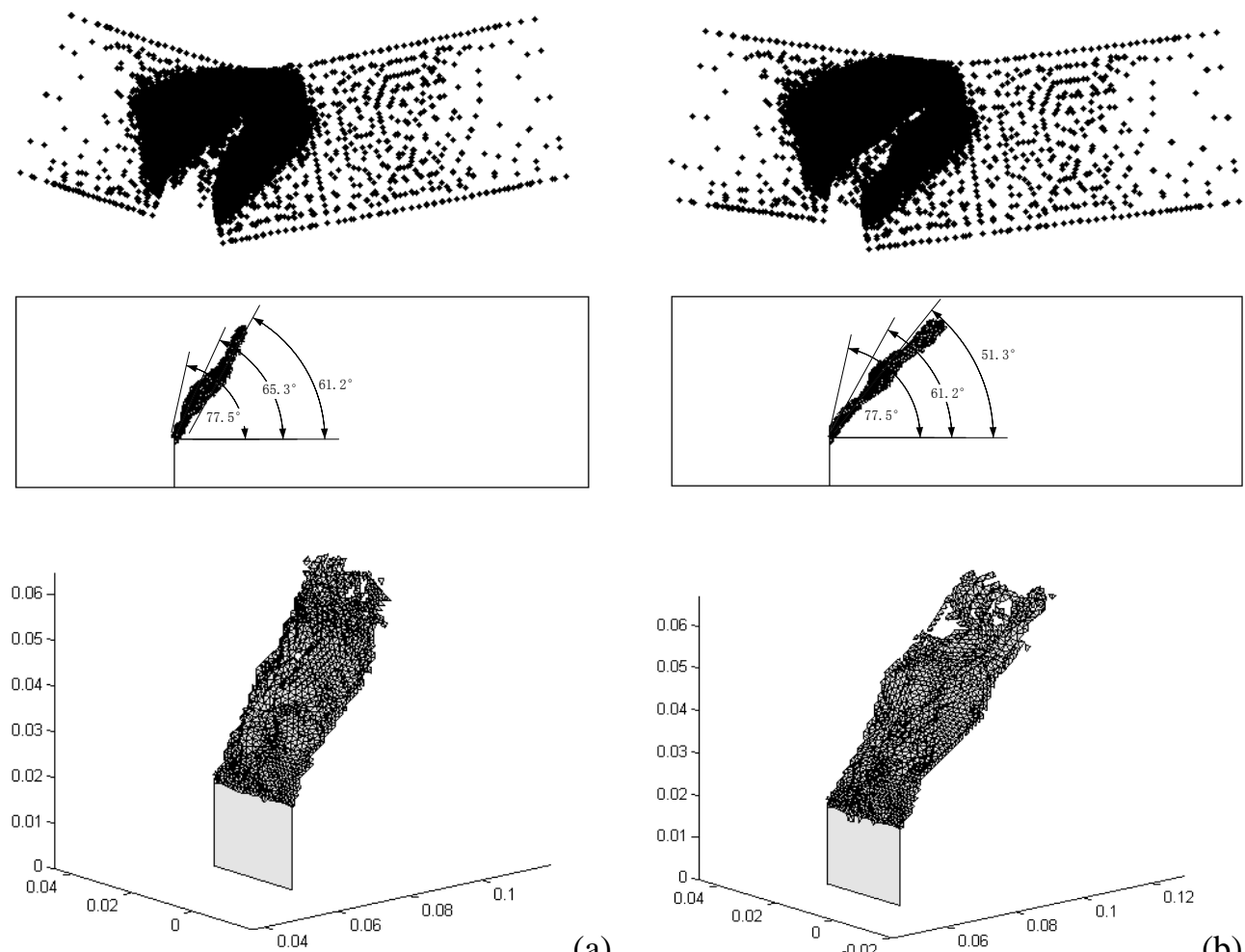

(a)

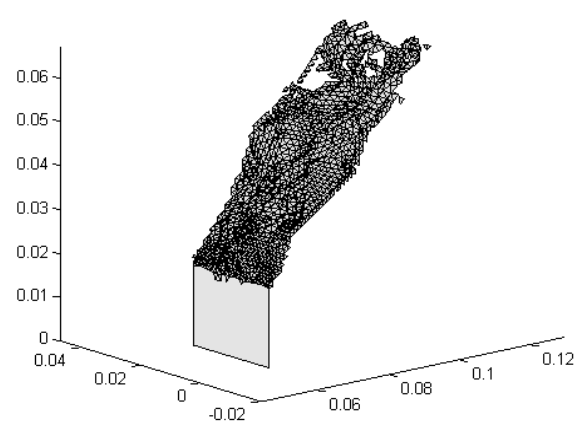

(b)

Fig.13 Simulated fracture profiles and morphologies (a) Using the hyperelastic-bilinear bond potential; (b) Using the conventional hyperelastic bond potential (in each subfigure, the upper one is the nodes in the last configuration where the displacements are magnified 350 times; the middle one is the extended fracture profile; the bottom one is the extended crack surface).

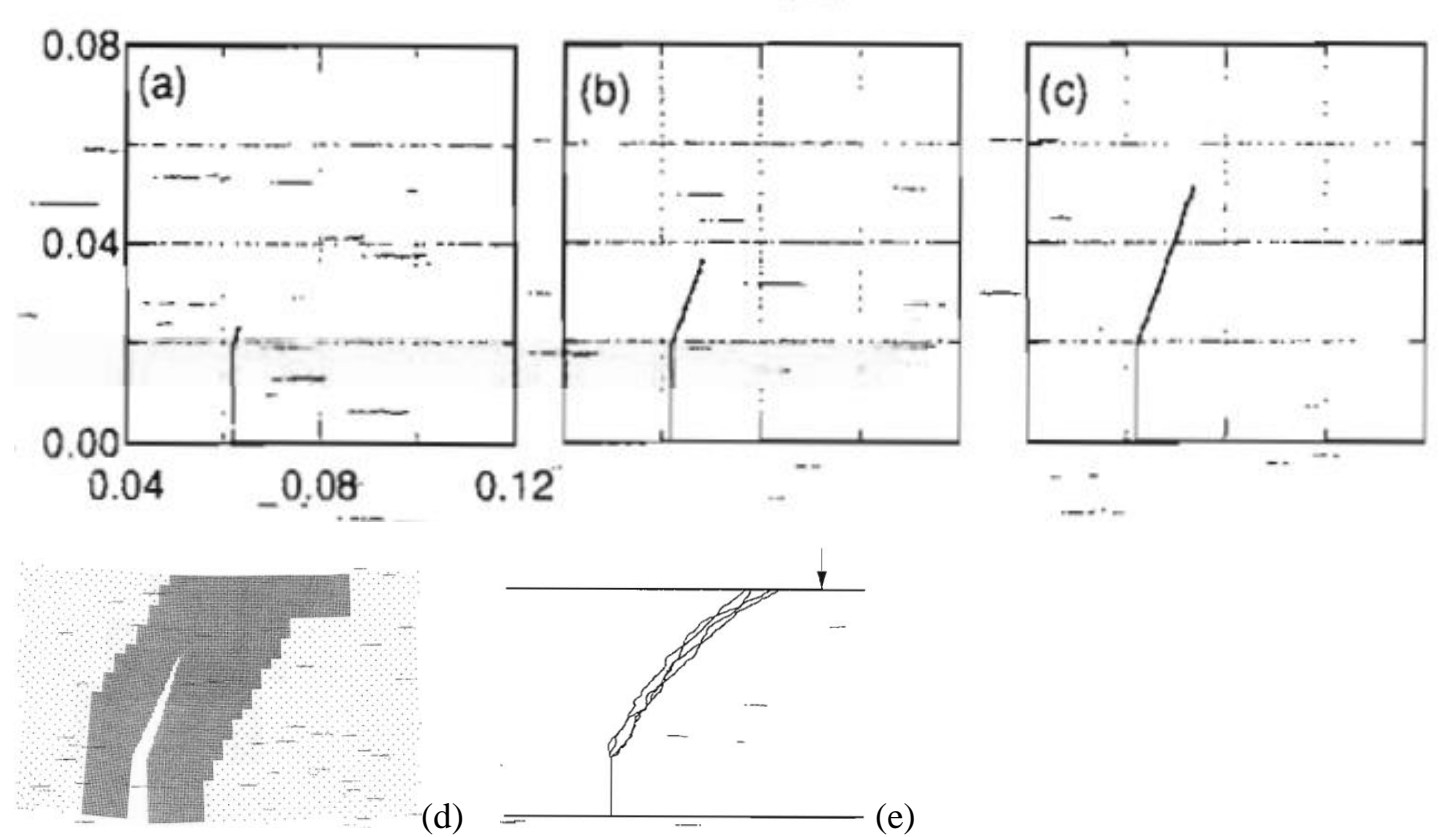

Fig.14 The simulated crack path in[43](Figures 11-13 in [43]) for John's [44] experiments at (a) $t=720.3 \mu \mathrm{s}$; (b) $t=842.8 \mu \mathrm{s}$; (c) $t=980.0 \mu \mathrm{s}$; (d) fracture profile; (e) crack paths emanating from the initial vertical notch reported in [44]. 


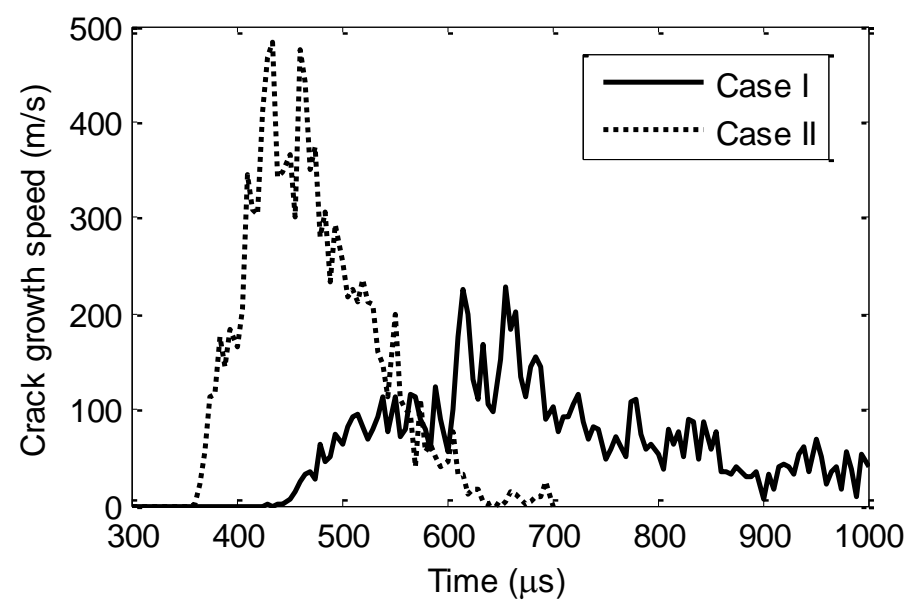

(a)

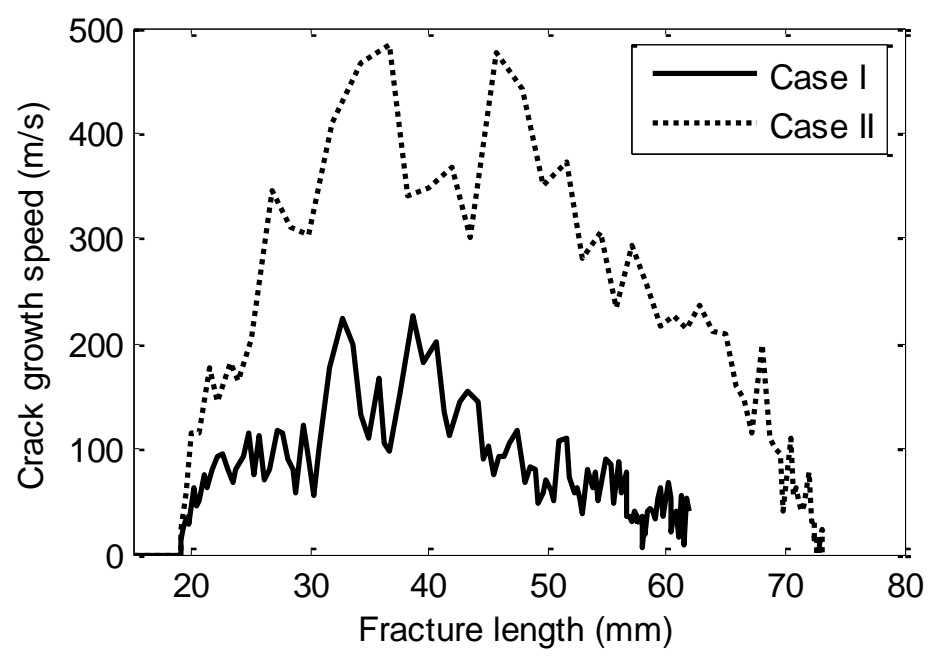

Fig.15 Simulated crack speed (a) Crack speed versus time; (b) Crack speed versus fracture length. (the crack speed is averaged over the time interval 5.0 $\mu \mathrm{s}$ ).

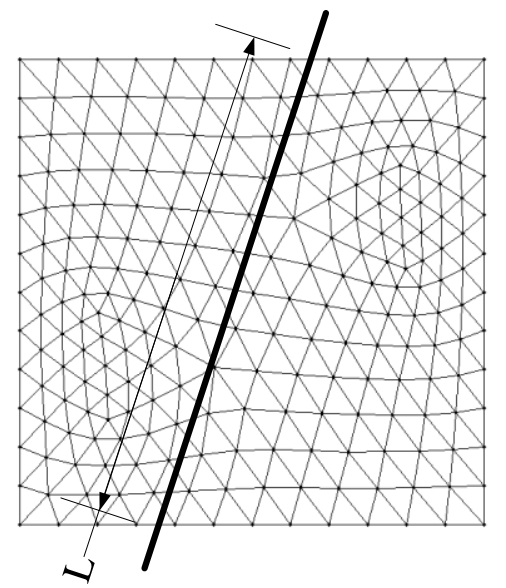

Fig.A1 A straight line cut through a square composed of irregular triangular unit cells. 


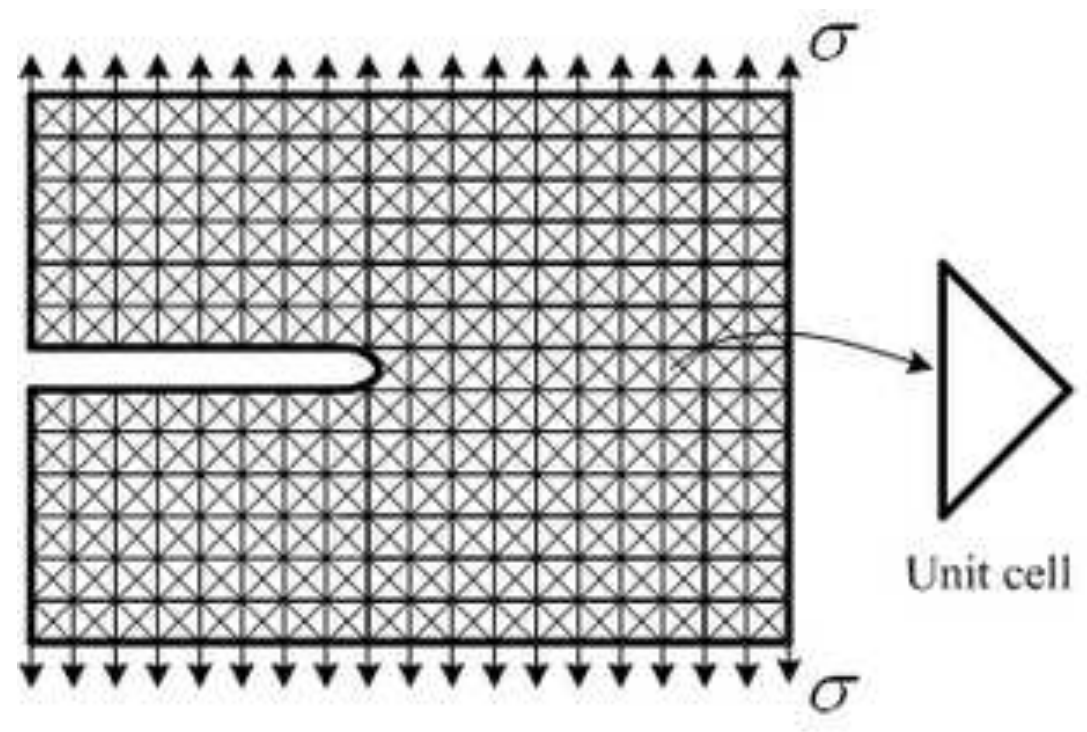

(a)

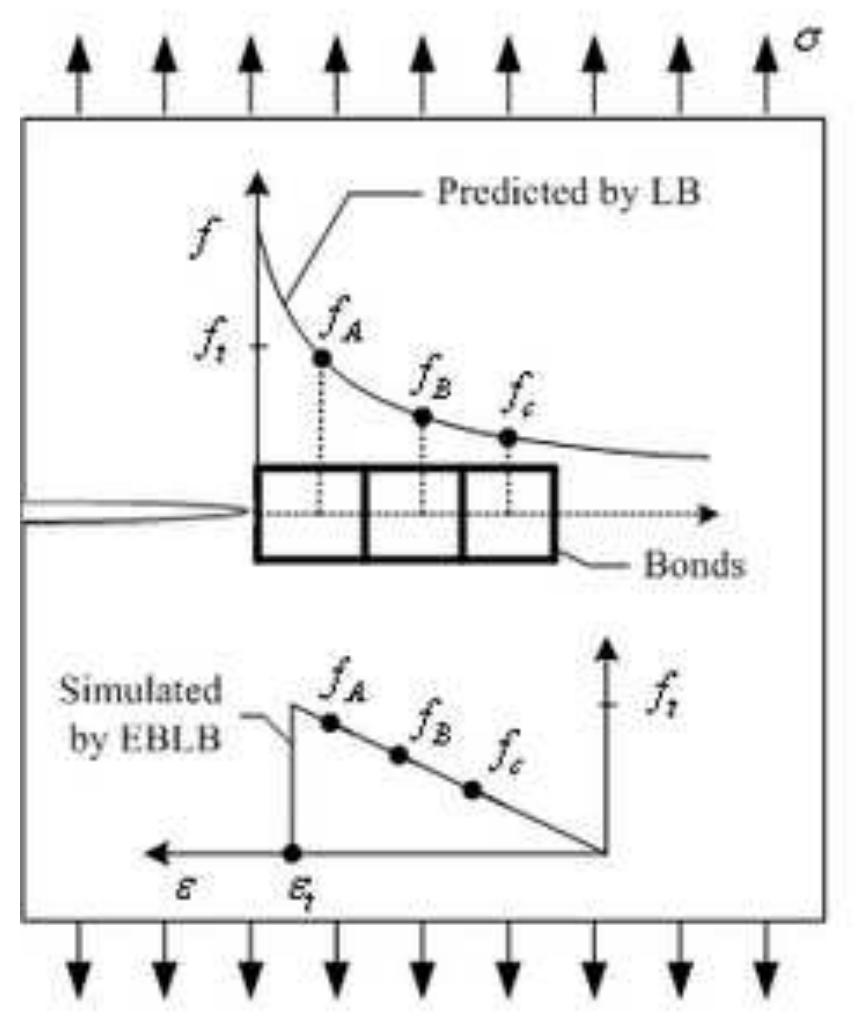

(c)

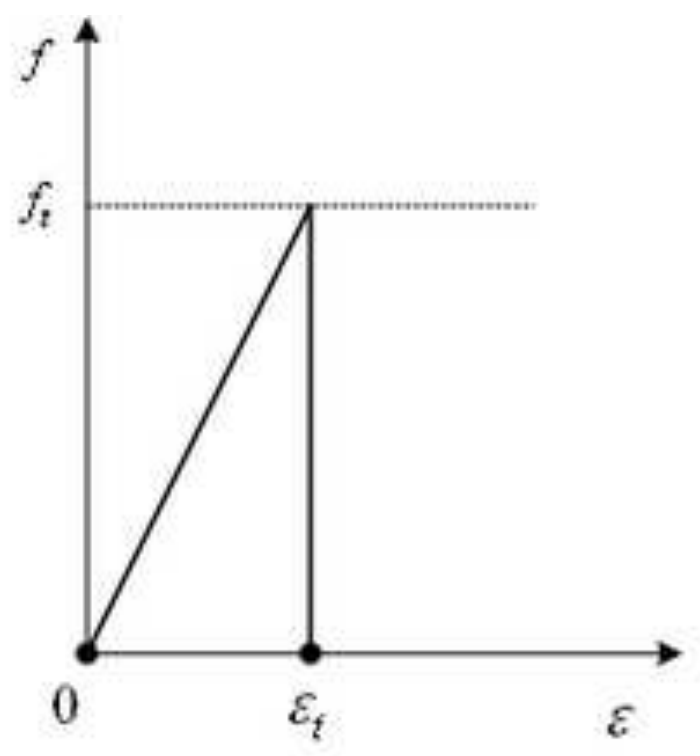

(b)

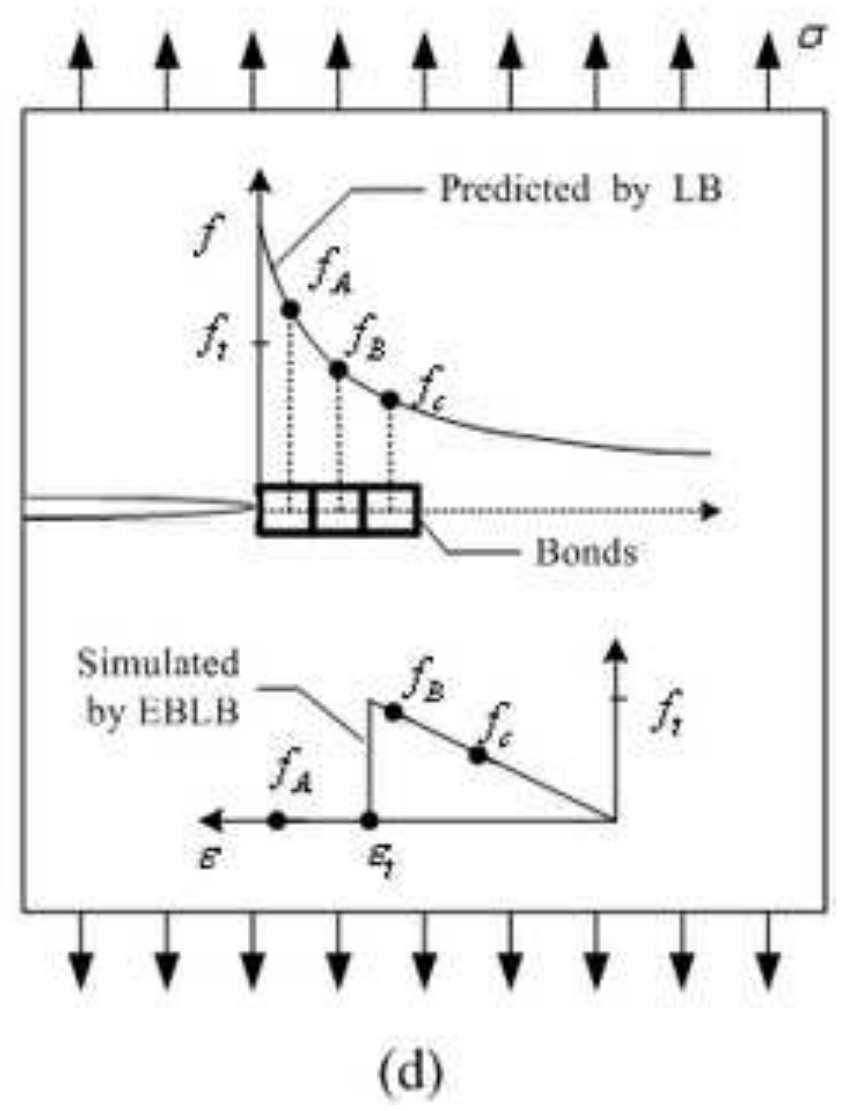




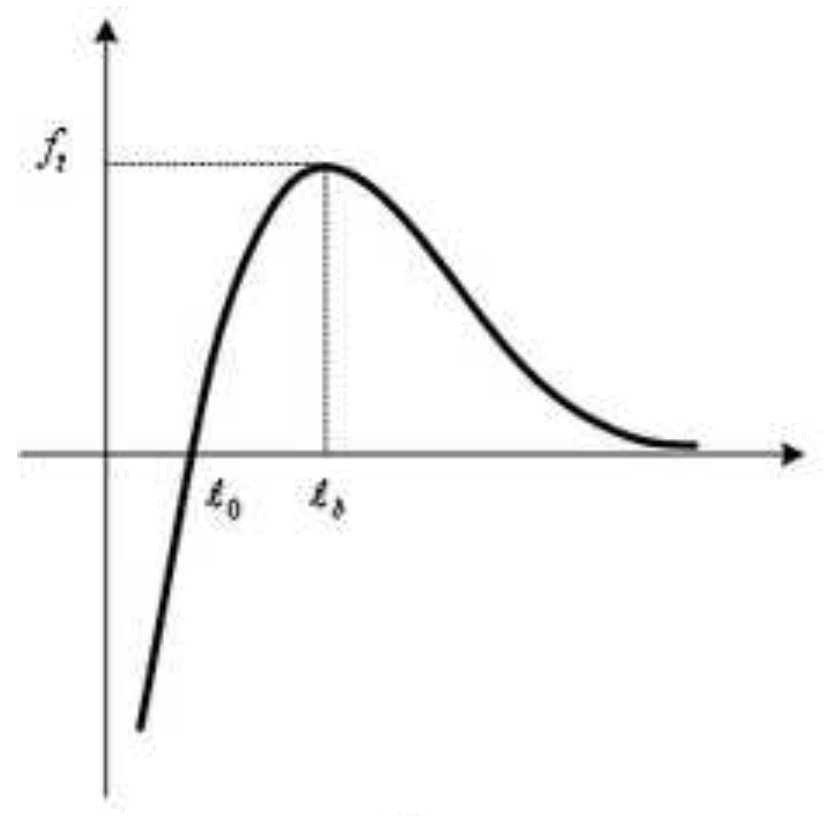

(a)

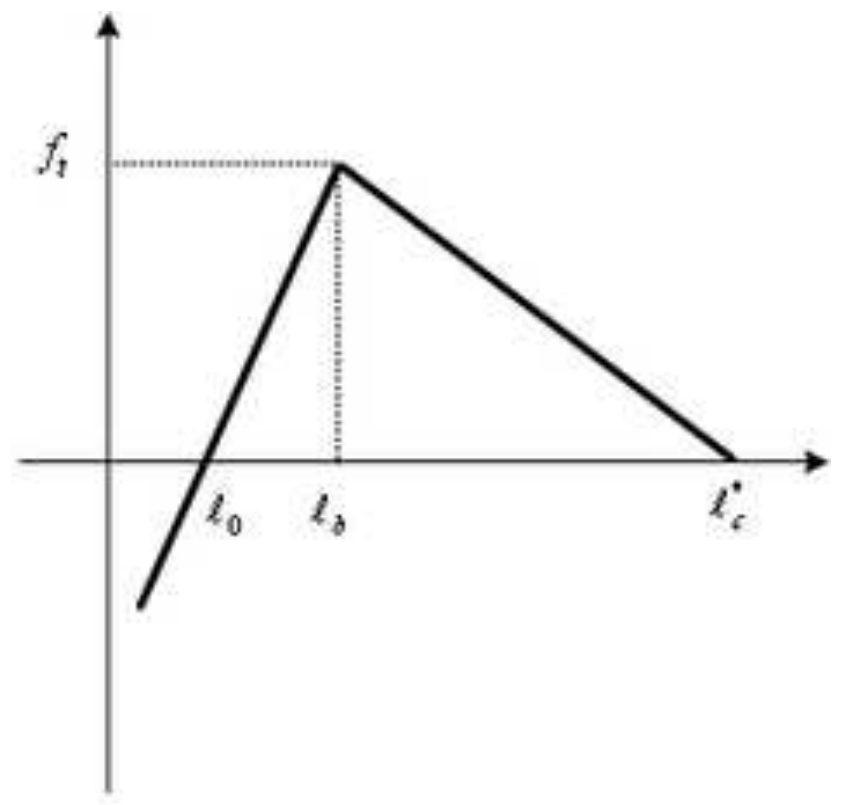

(b)

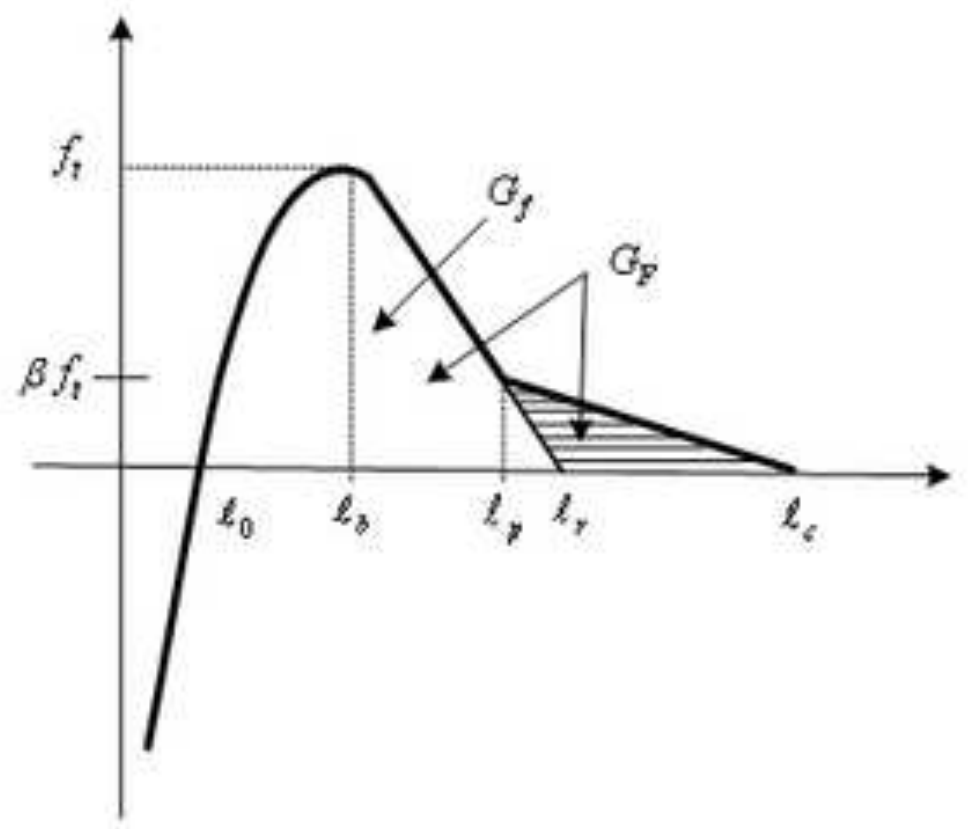

(c) 


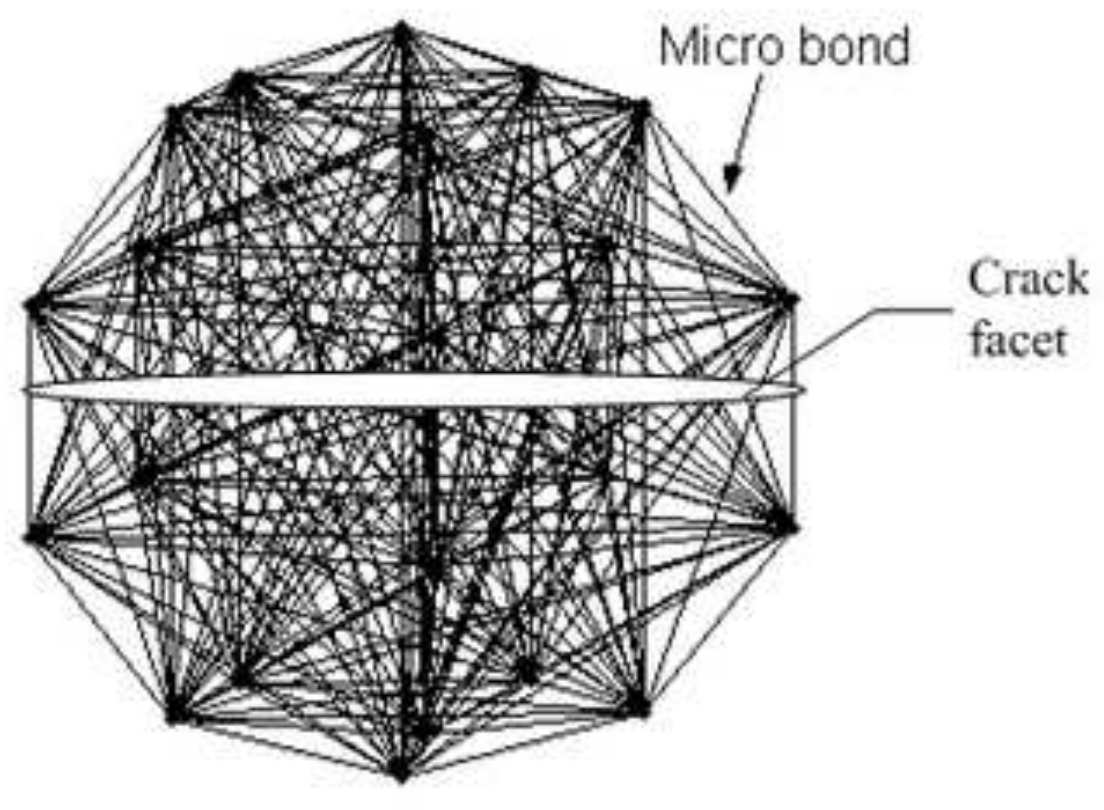

(a)

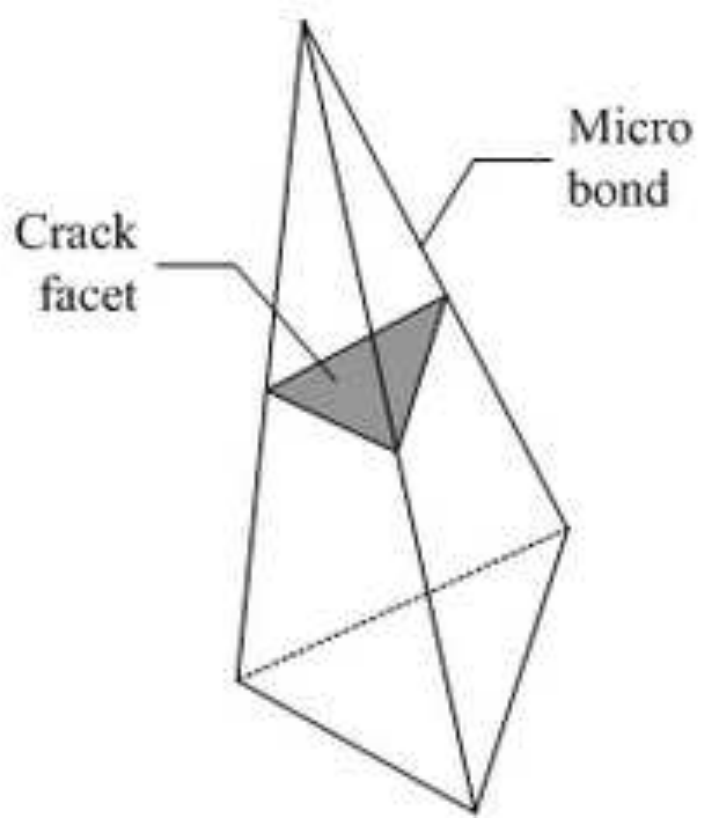

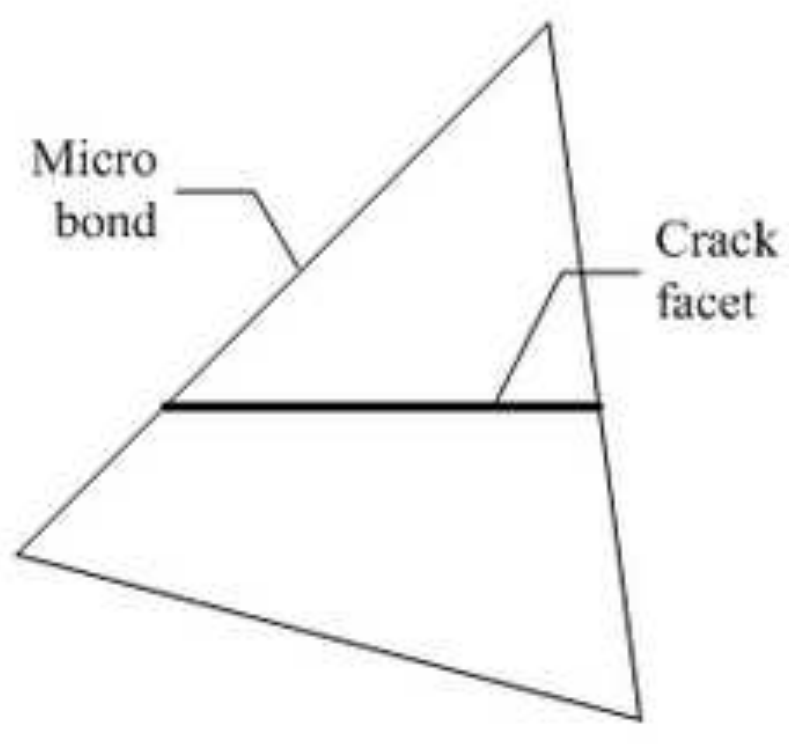

(b)

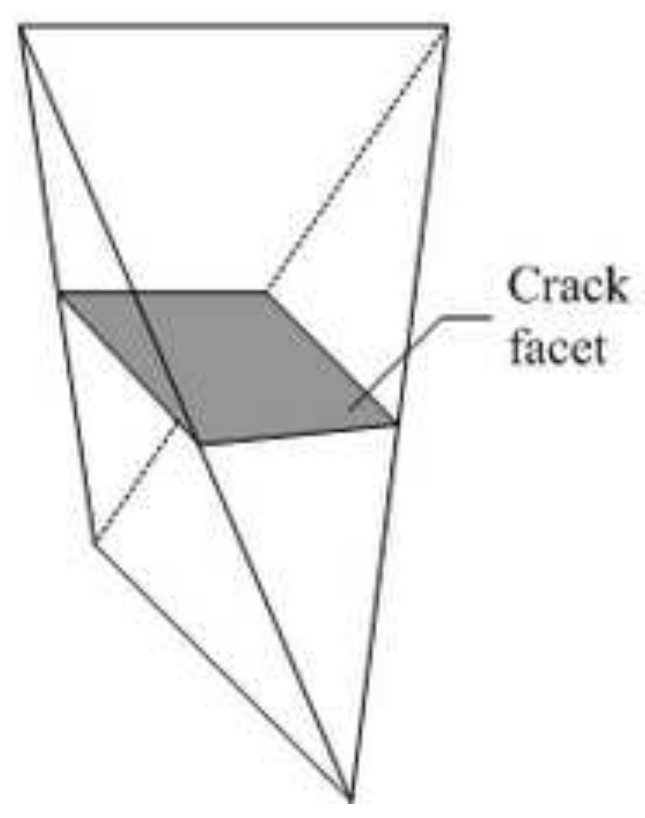

(c) 


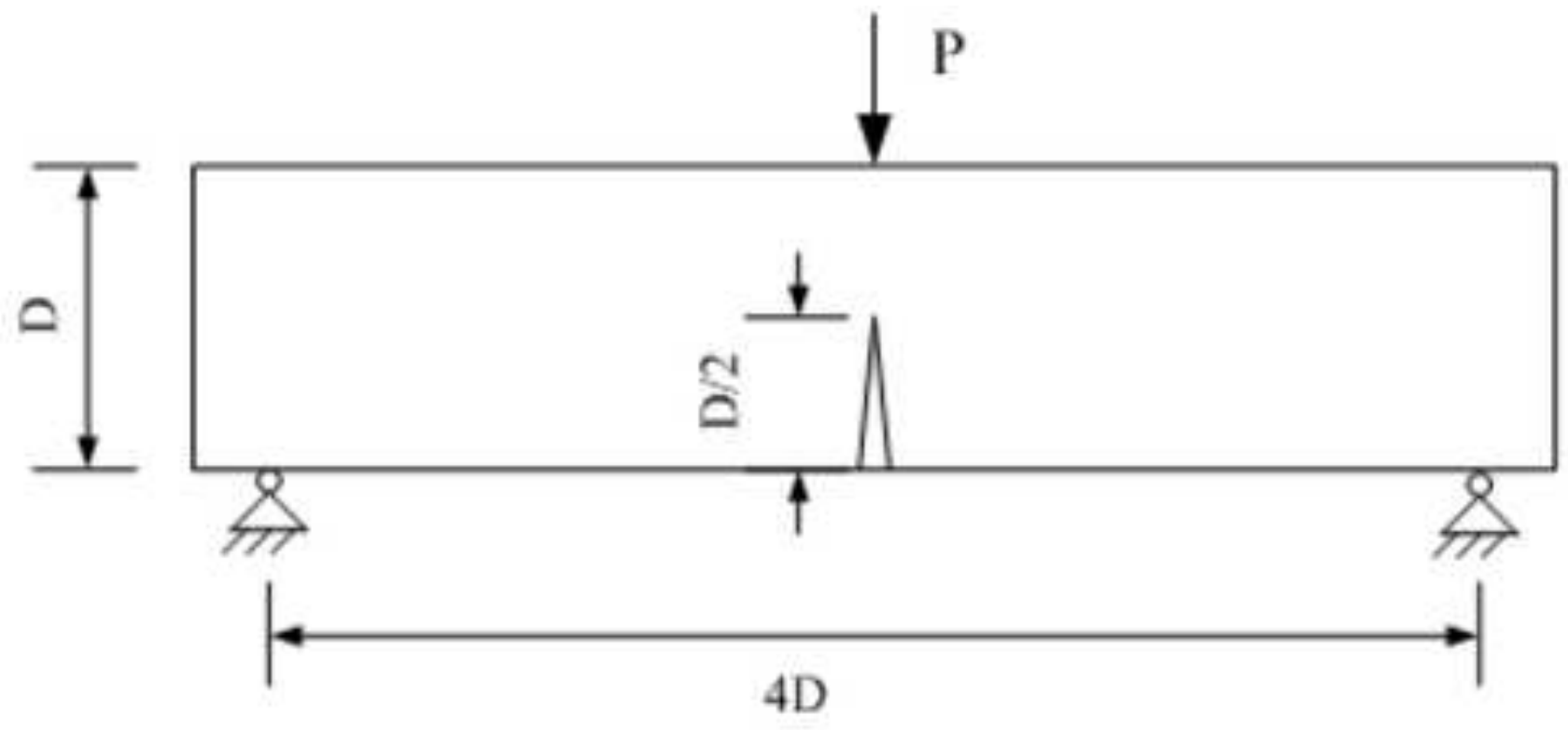

(a)

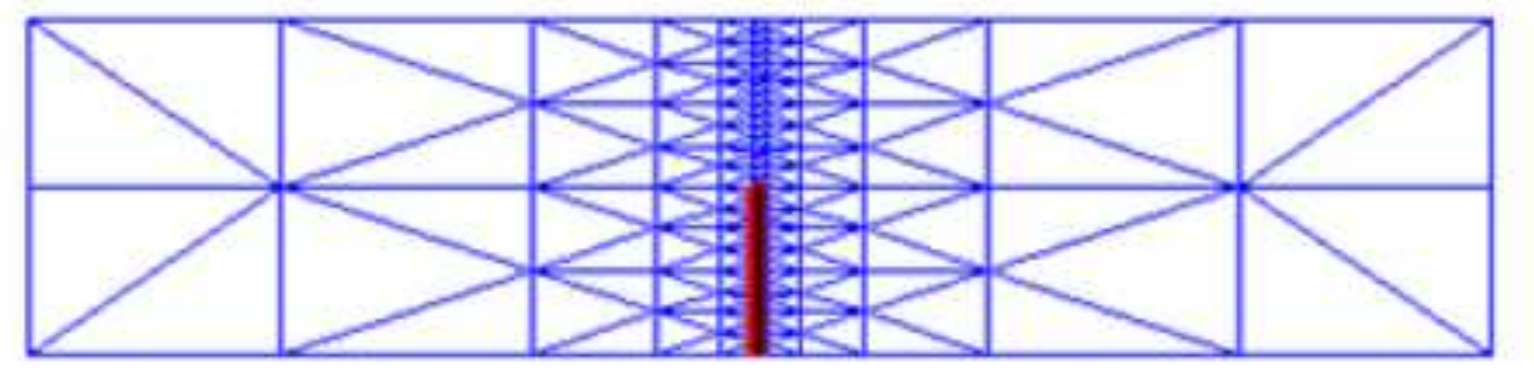

(b)

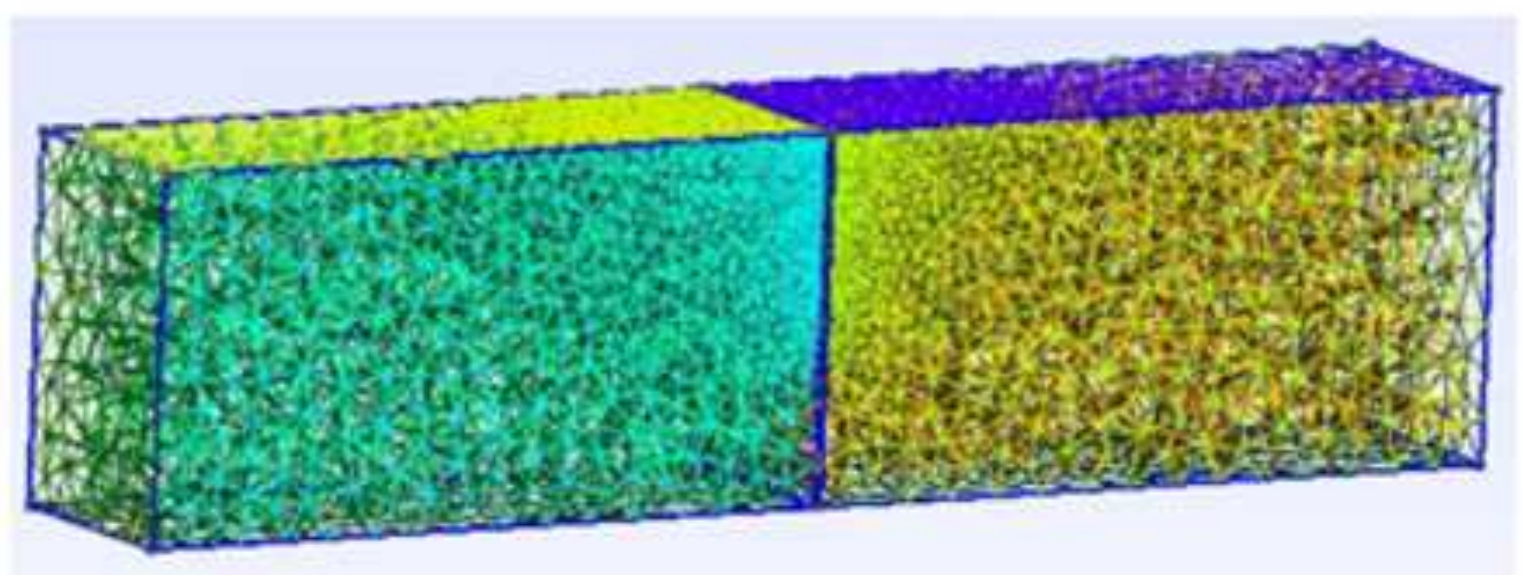

(c)

Figure 4 


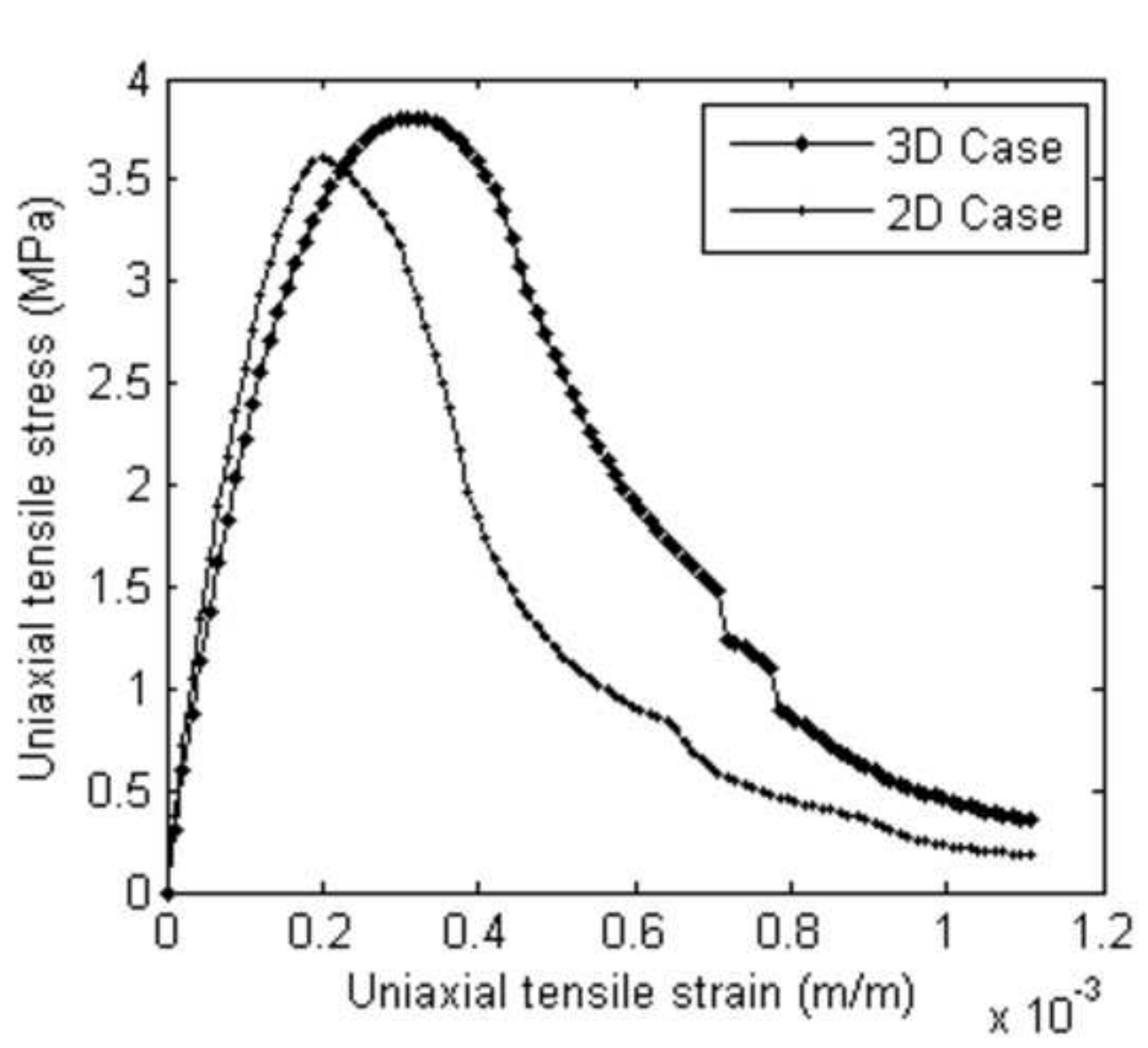




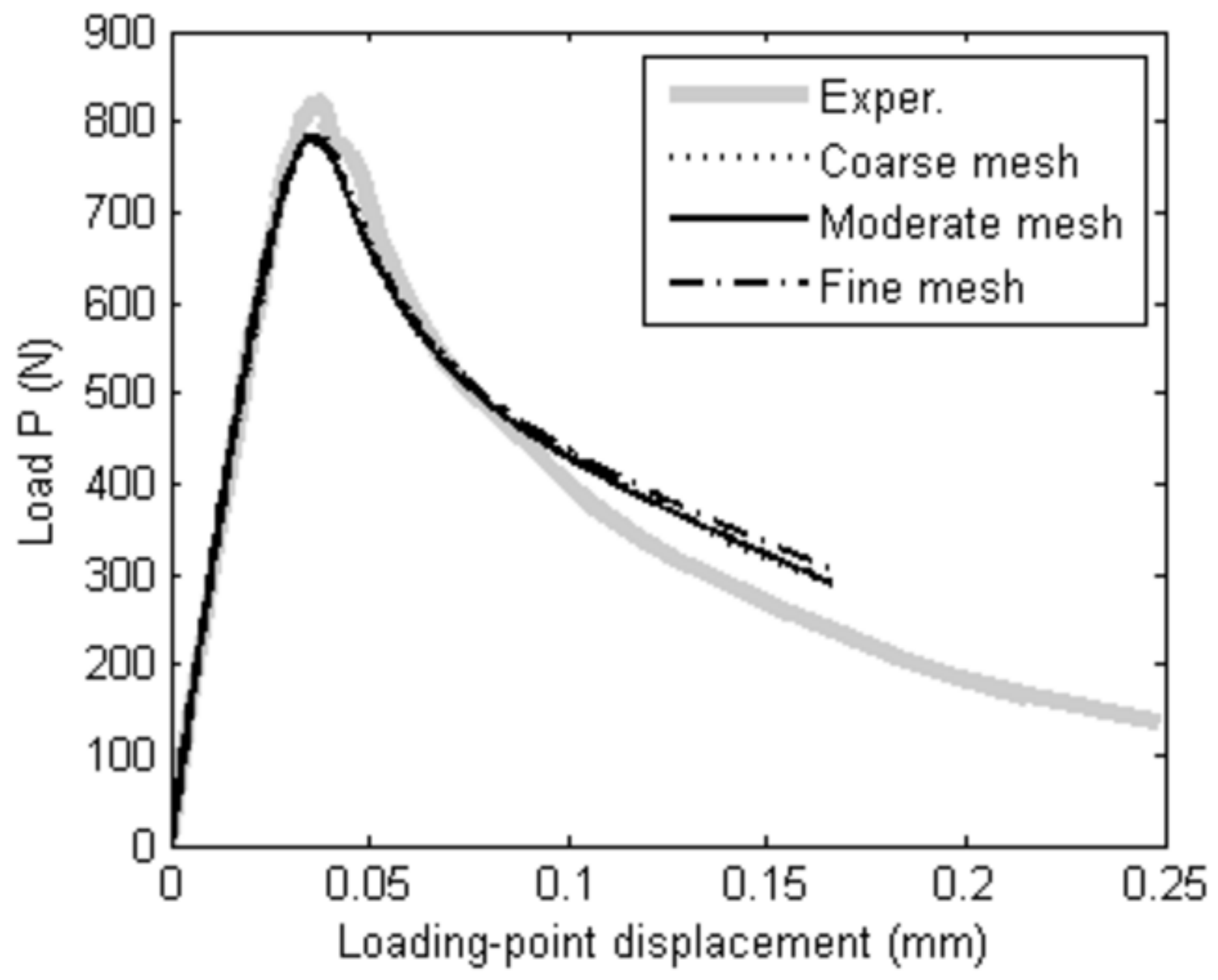




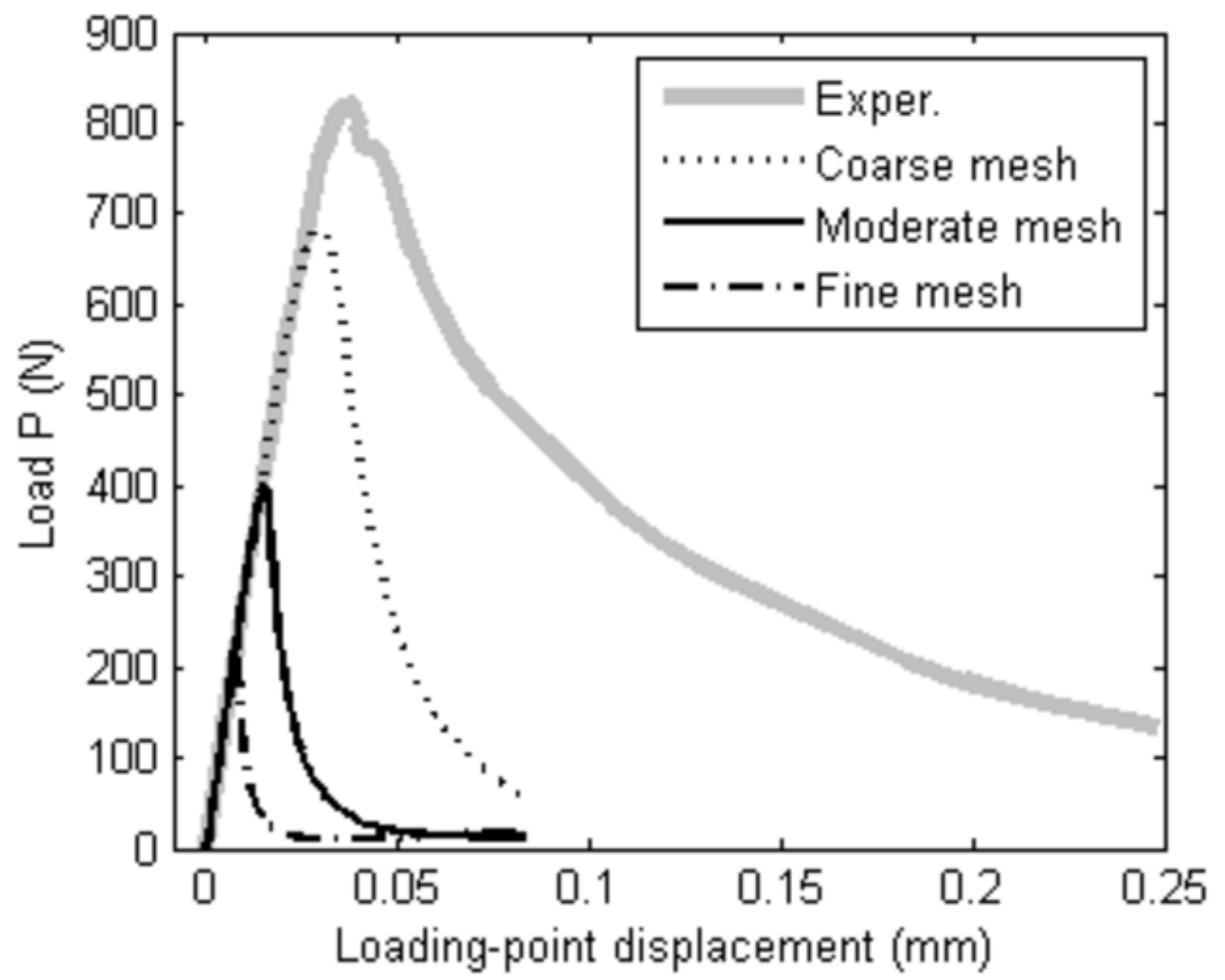




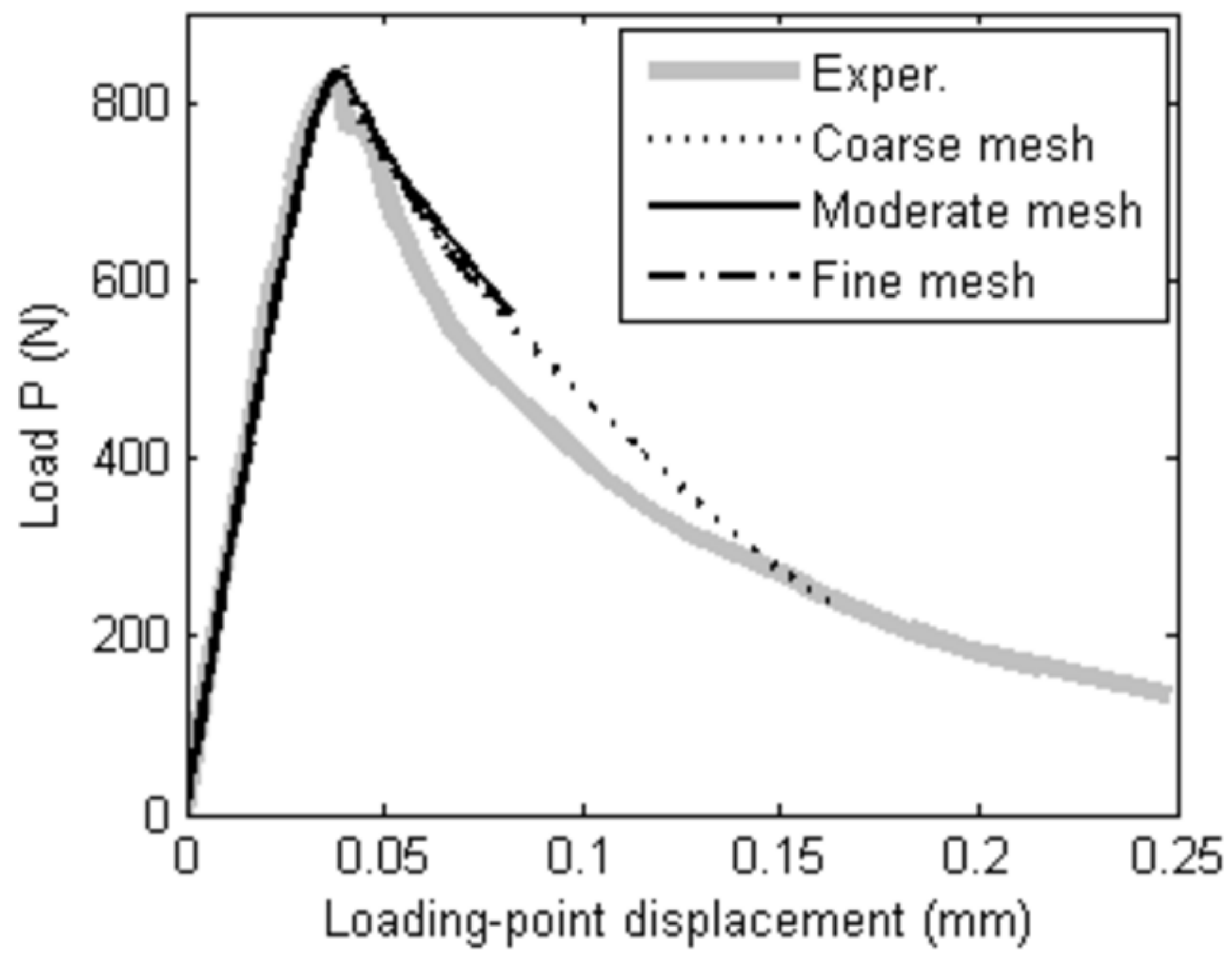




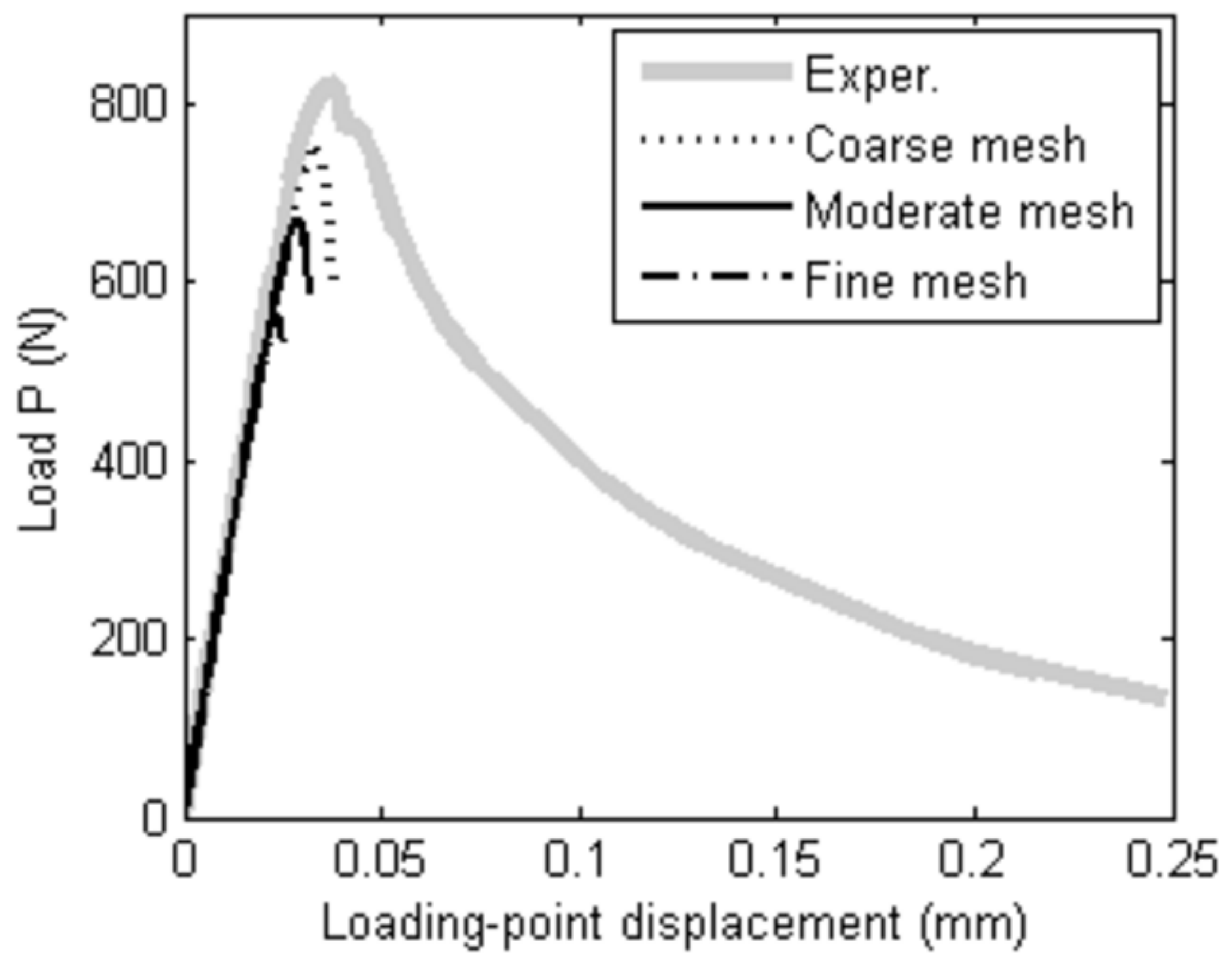




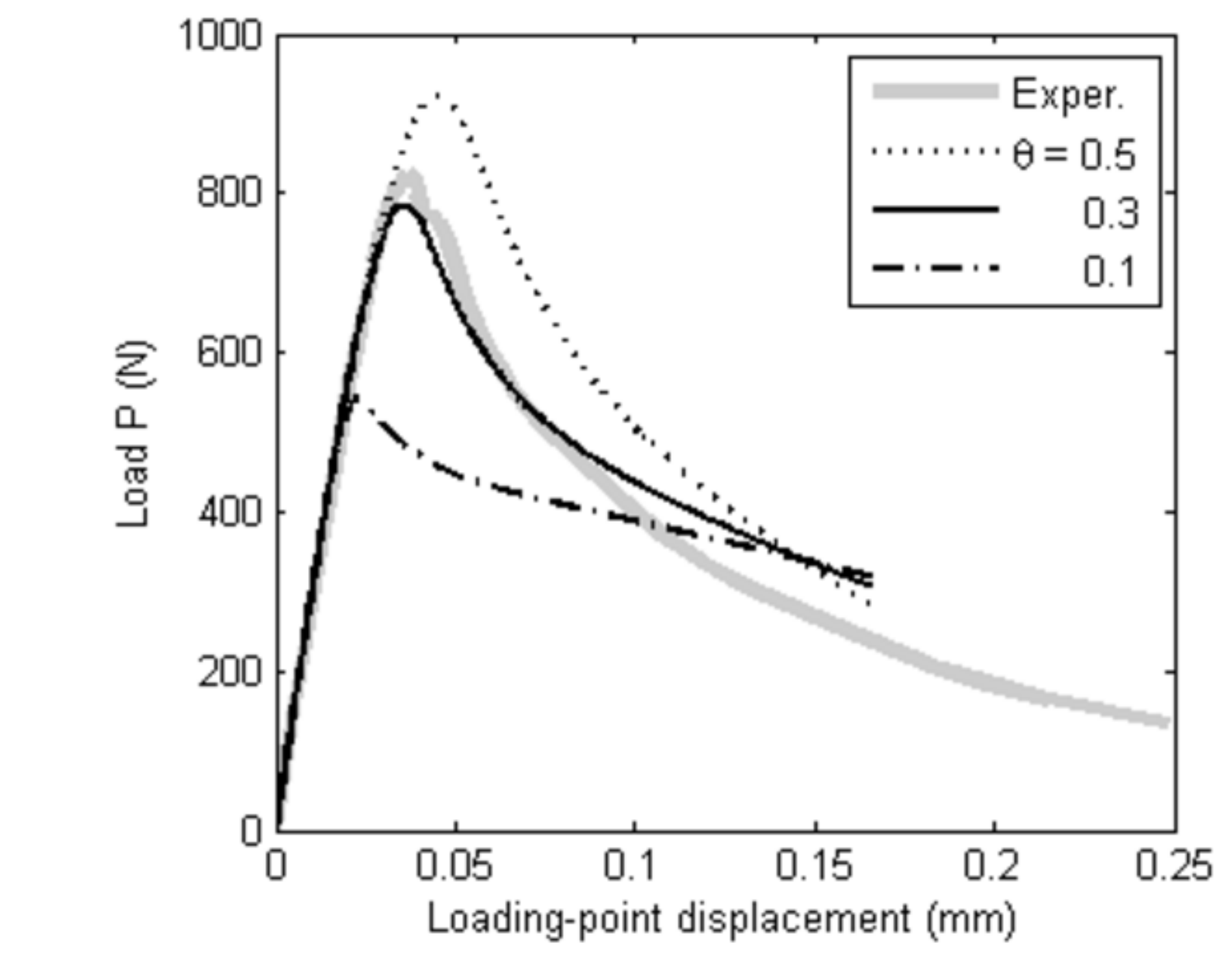




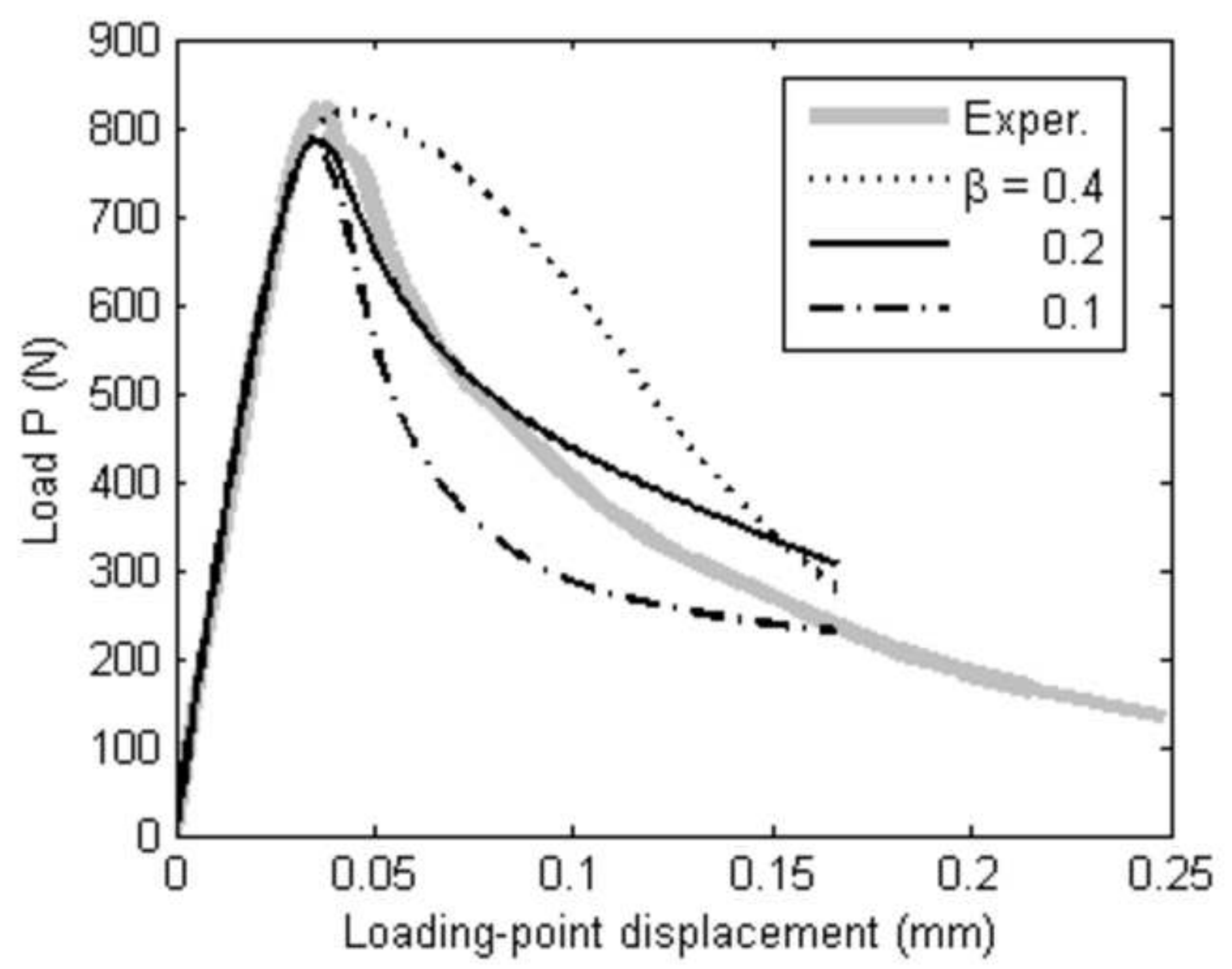




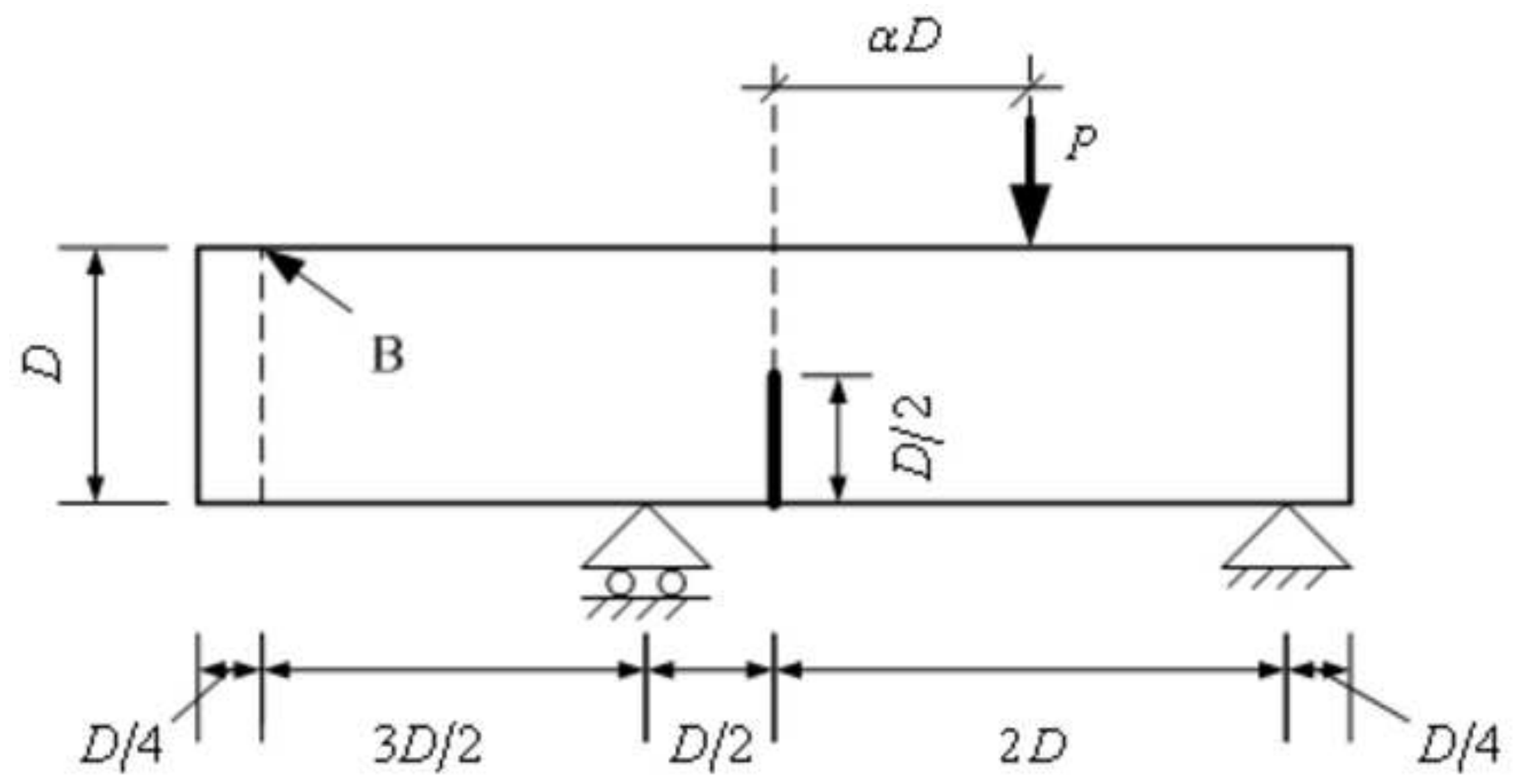

(a)

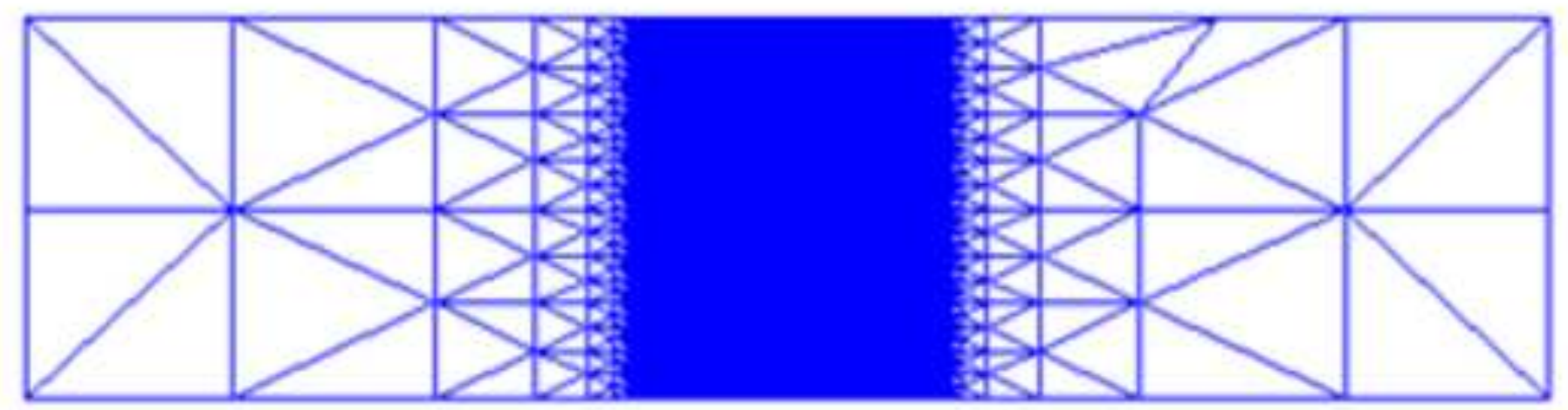

(b) 


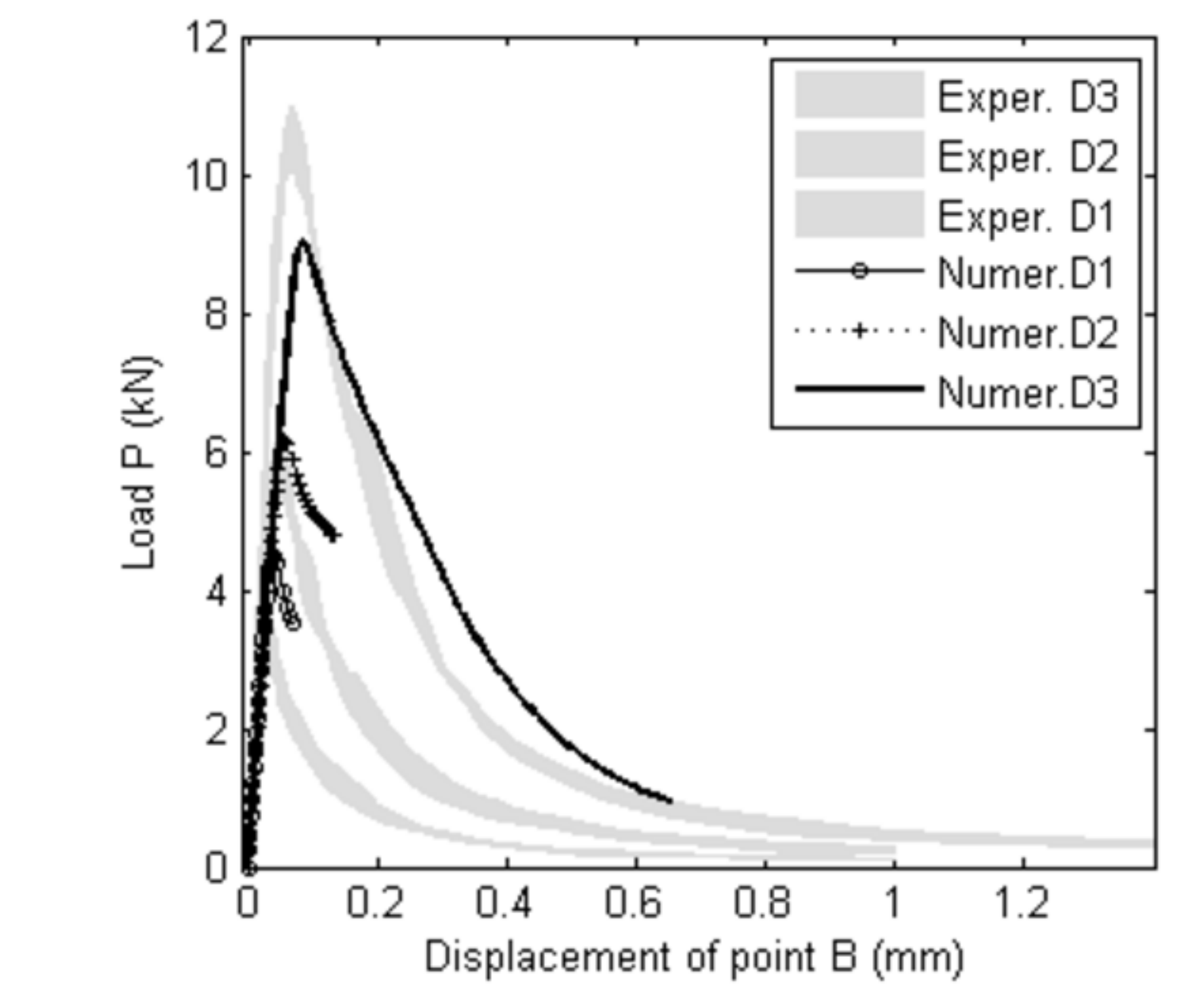




\section{$\downarrow \downarrow \downarrow \downarrow^{V_{0}}$}
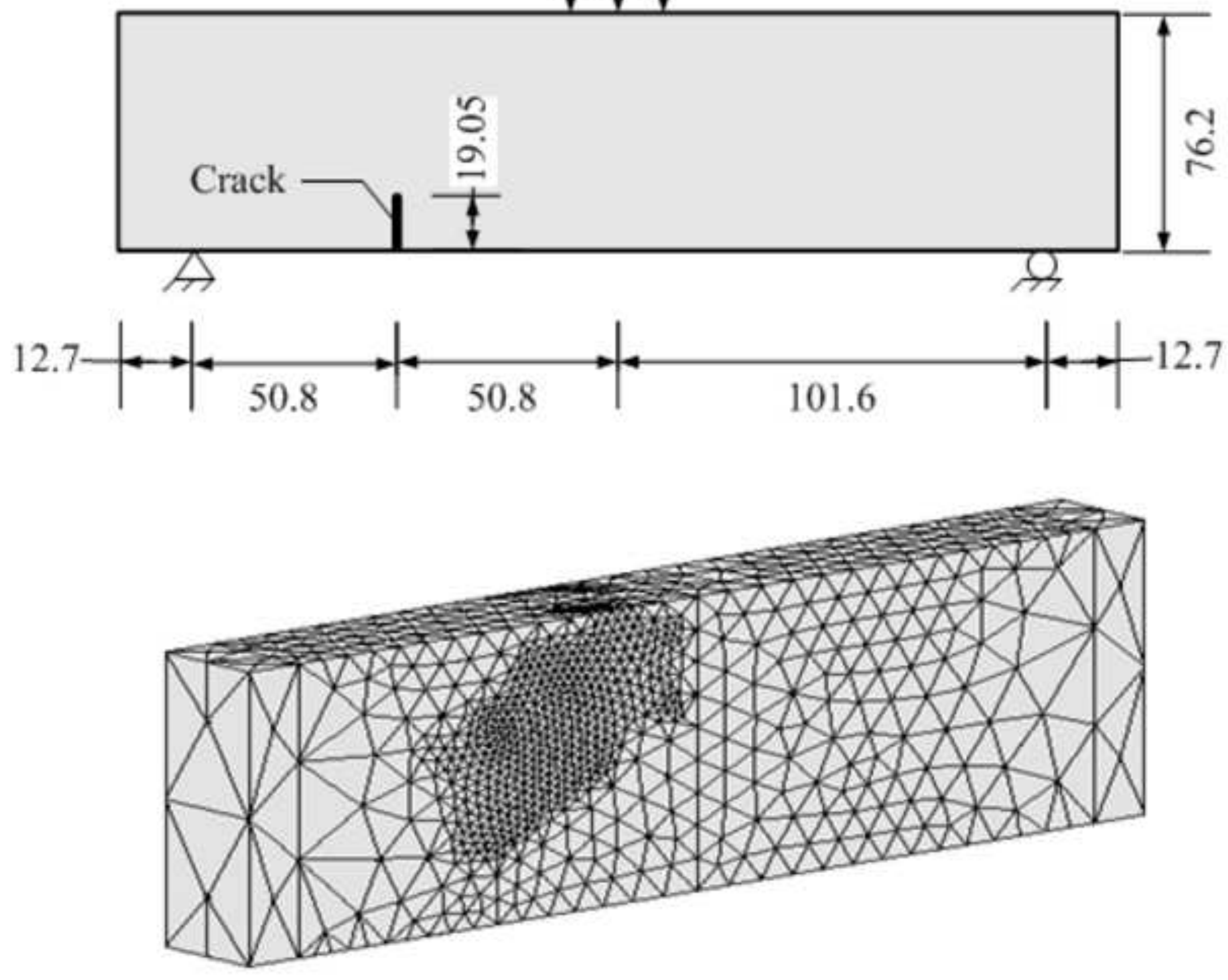


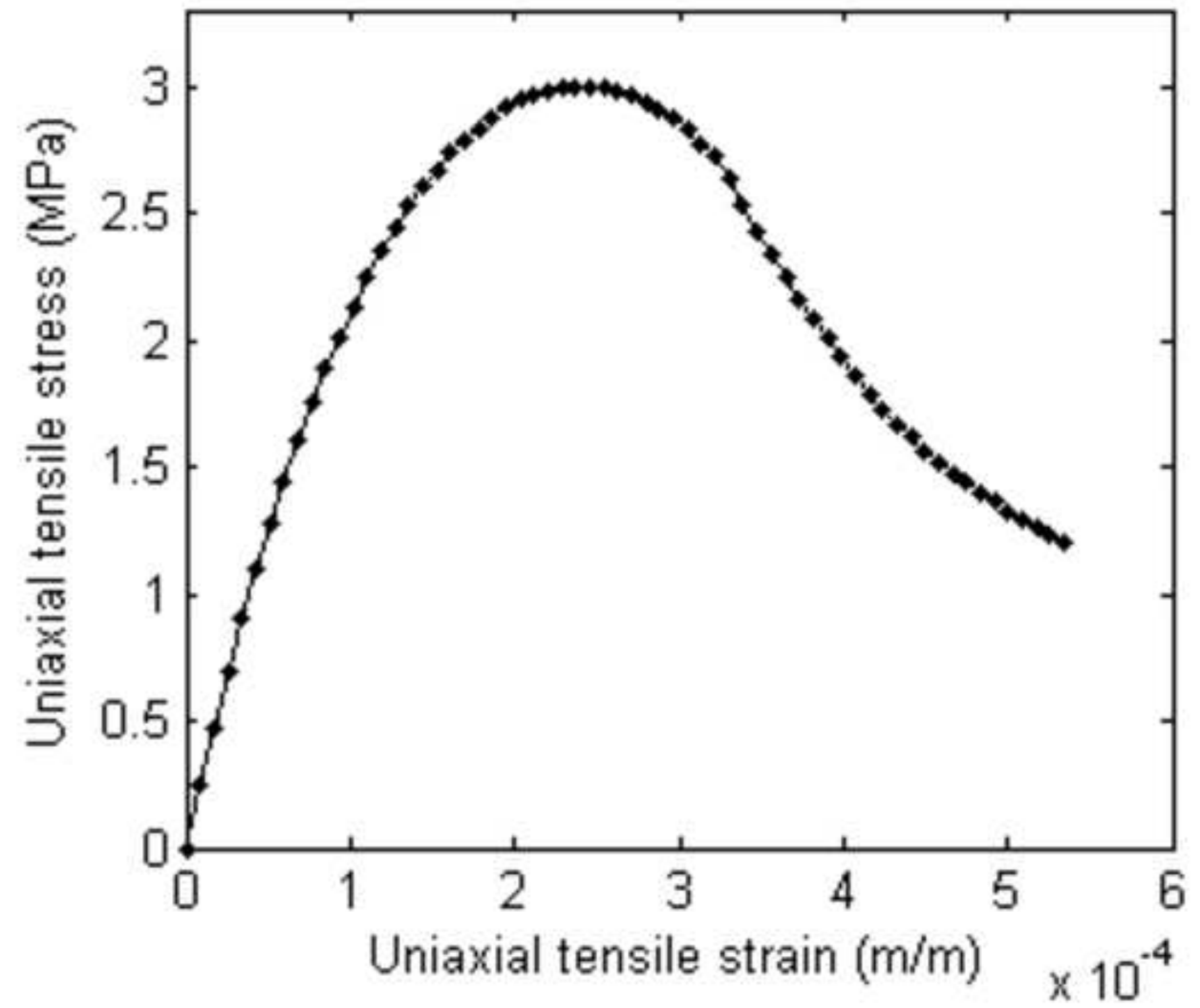


Figure 13b
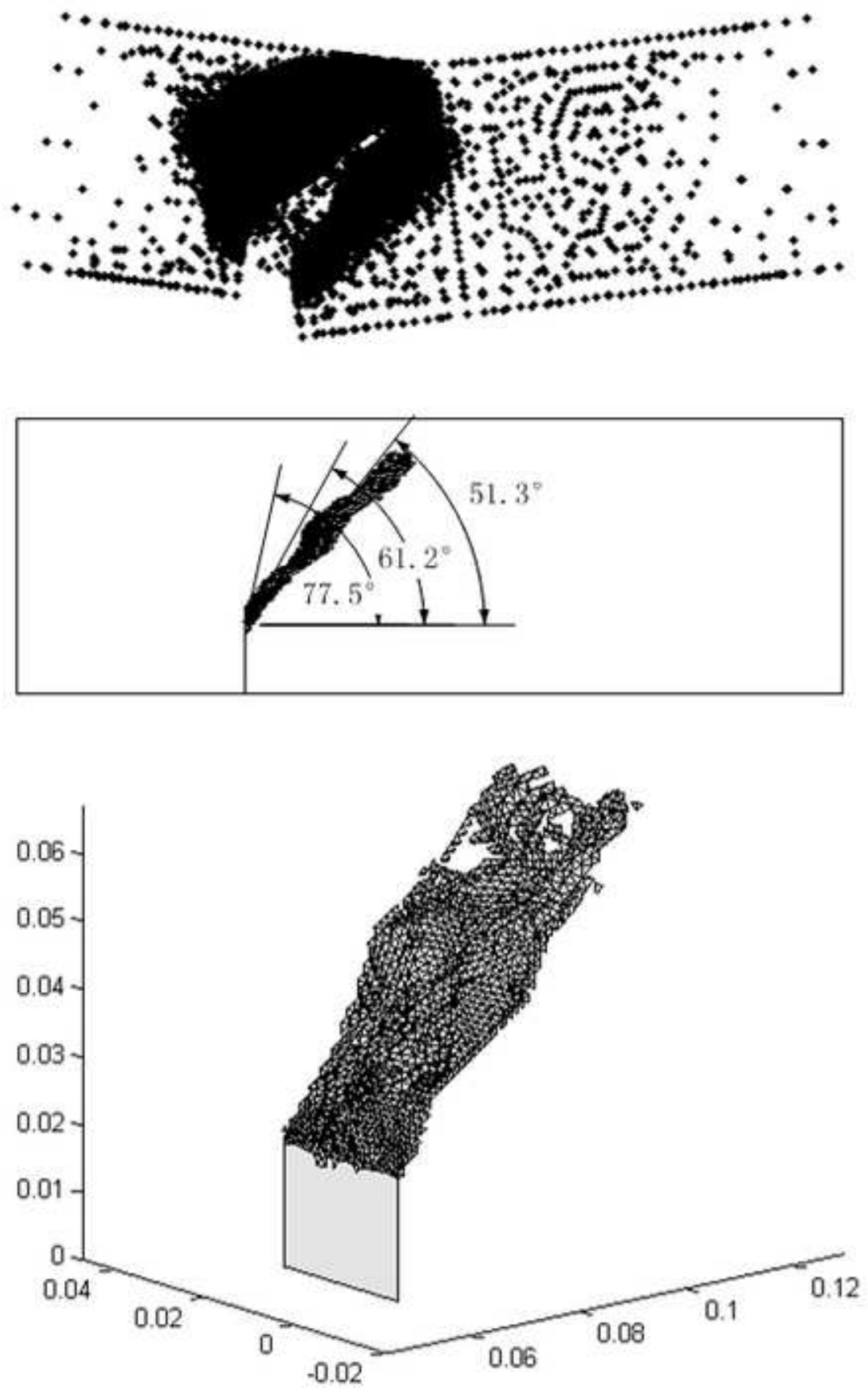


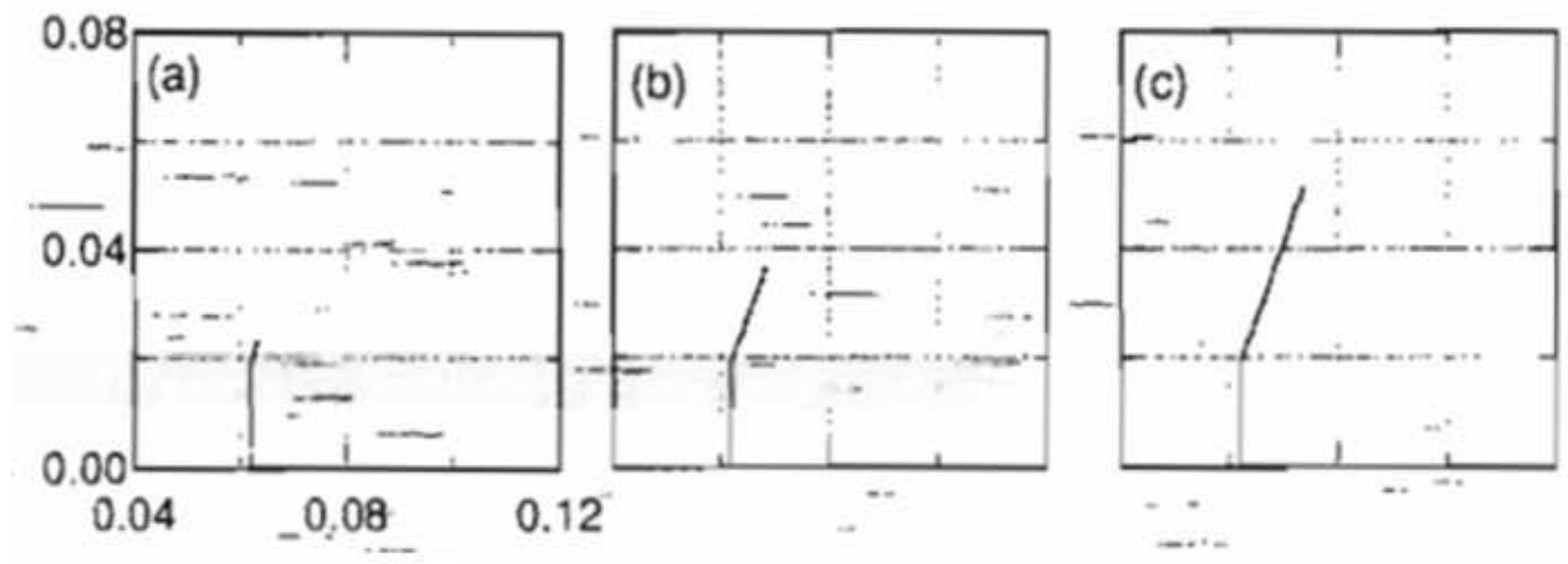

(d)

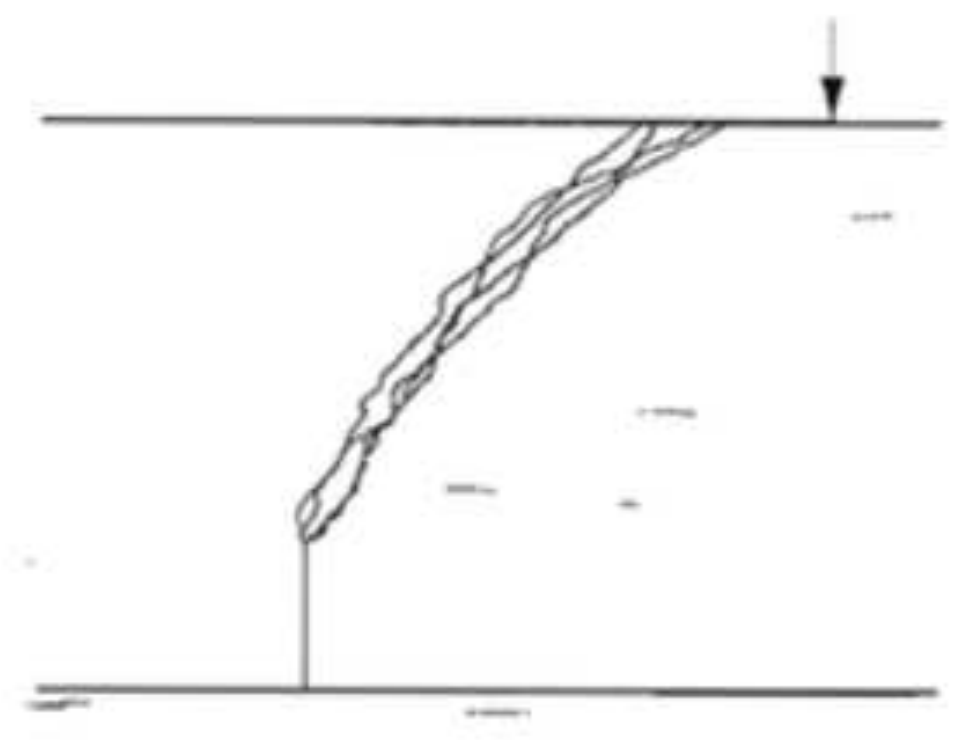

(e) 


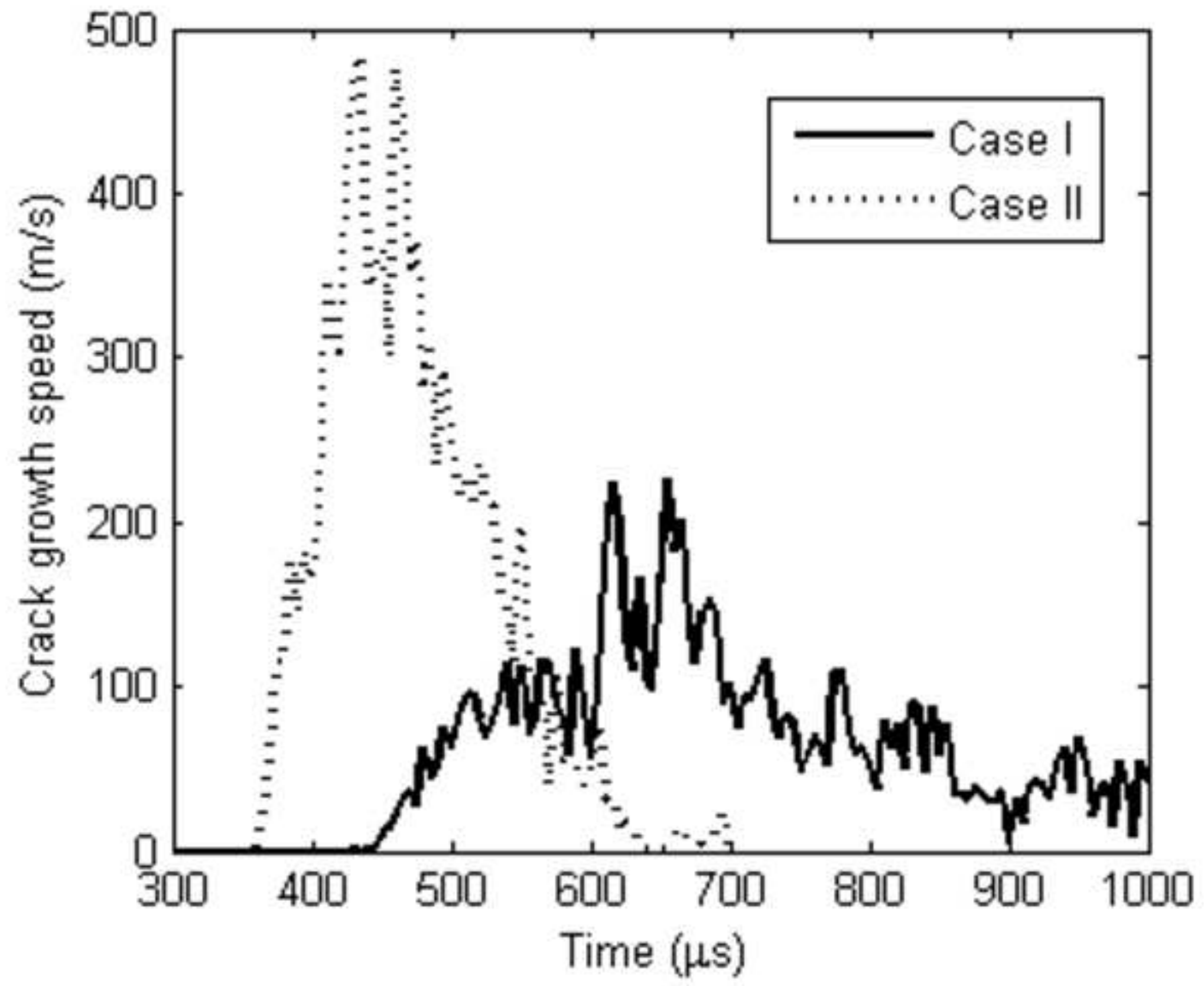




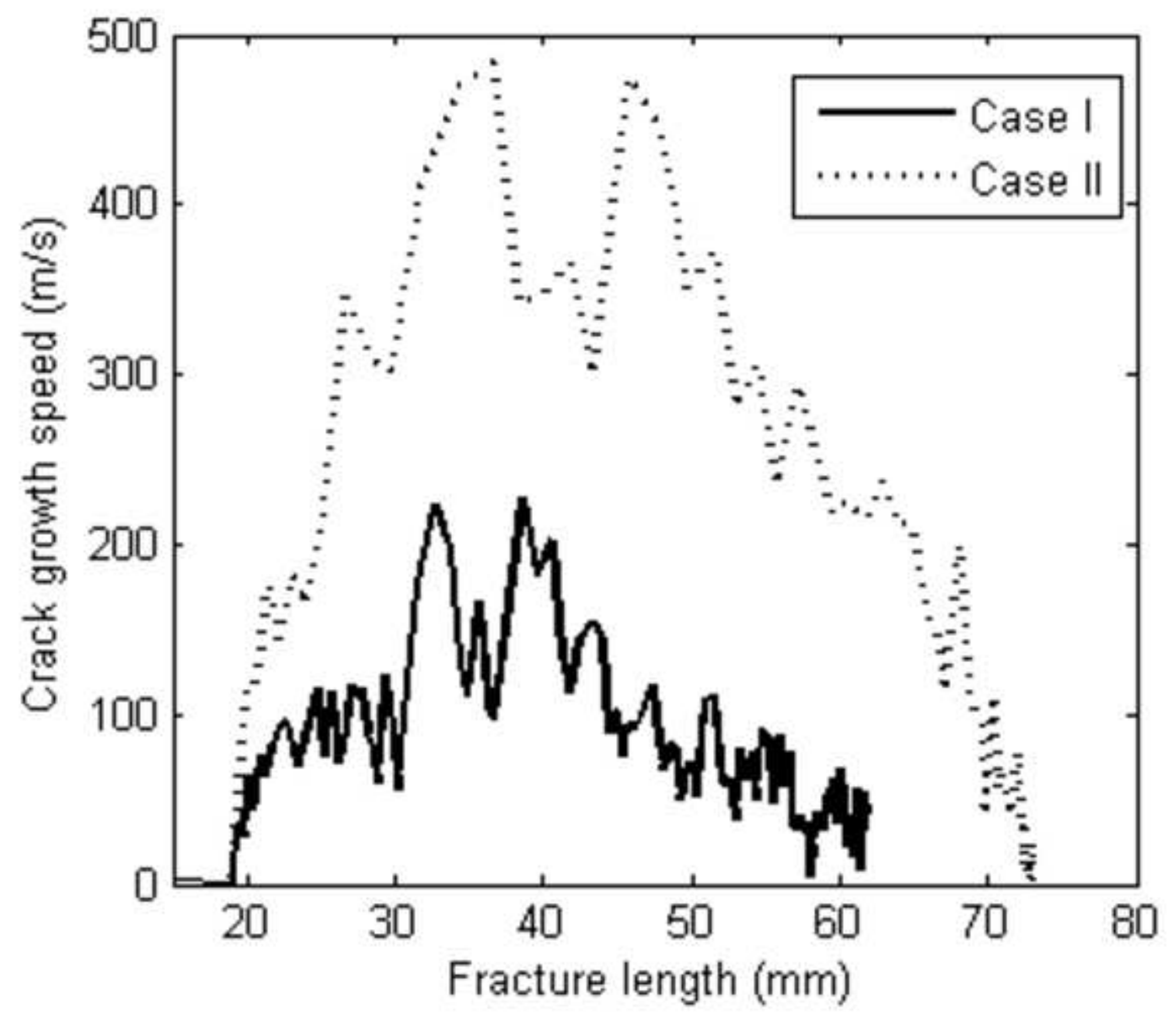




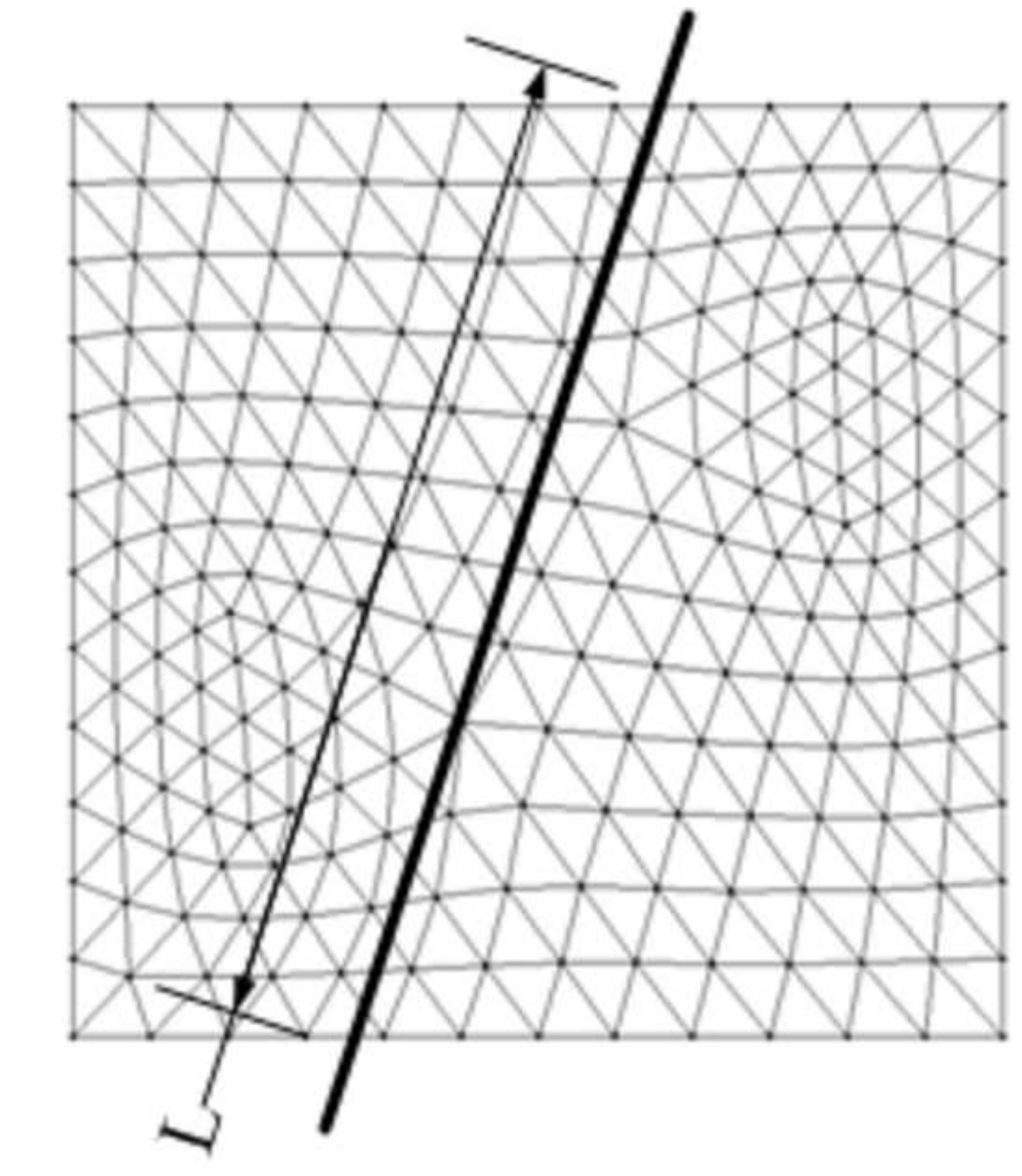

Figure A1

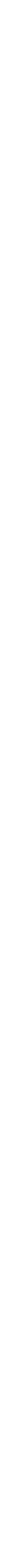

.

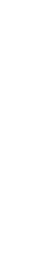

